\title{
Truncated netrin-1 contributes to pathological vascular permeability in diabetic retinopathy
}

\author{
Khalil Miloudi, ${ }^{1,2}$ François Binet, ${ }^{2}$ Ariel Wilson, ${ }^{3}$ Agustin Cerani, ${ }^{4}$ Malika Oubaha, ${ }^{4}$ Catherine Menard, ${ }^{4}$ \\ Sullivan Henriques, ${ }^{2}$ Gaelle Mawambo, ${ }^{4}$ Agnieszka Dejda, ${ }^{4}$ Phuong Trang Nguyen, ${ }^{5}$ Flavio A. Rezende, ${ }^{2}$ \\ Steve Bourgault, ${ }^{5}$ Timothy E. Kennedy, ${ }^{1}$ and Przemyslaw Sapieha ${ }^{1,2,4}$ \\ 'Department of Neurology and Neurosurgery, McGill University, Montreal, Quebec, Canada. ${ }^{2}$ Department of Ophthalmology, Maisonneuve-Rosemont Hospital Research Centre, University of Montreal, \\ Montreal, Quebec, Canada. '3epartment of Engineering Physics, École Polytechnique de Montréal, Laser Processing and Plasmonics Laboratory, Montreal, Quebec, Canada. \\ ${ }^{4}$ Department of Biochemistry, Maisonneuve-Rosemont Hospital Research Centre, University of Montreal, Montreal, Quebec, Canada. ${ }^{5}$ Department of Chemistry, \\ Université du Québec à Montréal, Montreal, Quebec, Canada.
}

\begin{abstract}
Diabetic retinopathy (DR) is a major complication of diabetes and a leading cause of blindness in the working-age population. Impaired blood-retinal barrier function leads to macular edema that is closely associated with the deterioration of central vision. We previously demonstrated that the neuronal guidance cue netrin-1 activates a program of reparative angiogenesis in microglia within the ischemic retina. Here, we provide evidence in both vitreous humor of diabetic patients and in retina of a murine model of diabetes that netrin- 1 is metabolized into a bioactive fragment corresponding to domains $\mathrm{VI}$ and $\mathrm{V}$ of the full-length molecule. In contrast to the protective effects of full-length netrin-1 on retinal microvasculature, the VI-V fragment promoted vascular permeability through the uncoordinated 5B (UNC5B) receptor. The collagenase matrix metalloprotease 9 (MMP-9), which is increased in patients with diabetic macular edema, was capable of cleaving netrin-1 into the VI-V fragment. Thus, MMP-9 may release netrin-1 fragments from the extracellular matrix and facilitate diffusion. Nonspecific inhibition of collagenases or selective inhibition of MMP-9 decreased pathological vascular permeability in a murine model of diabetic retinal edema. This study reveals that netrin-1 degradation products are capable of modulating vascular permeability, suggesting that these fragments are of potential therapeutic interest for the treatment of DR.
\end{abstract}

\section{Introduction}

Diabetic retinopathy (DR) is one of the most common complications of diabetes (1). DR develops in a protracted manner and can ultimately result in severe impairment of visual function. Initially, nonproliferative DR is associated with microvascular complications such as microaneurysms, intraretinal hemorrhages, and eventual capillary closure and degeneration. As the disease progresses, advanced stages may lead to proliferative diabetic retinopathy (PDR), characterized by growth of leaky, hemorrhaging pre-retinal neovessels and potential vitreal contraction and retinal detachment $(2,3)$.

Throughout the course of the disease, deterioration of the blood-retinal barrier (BRB) results in extravasation of fluids from permeable capillaries, leading to diabetic macular edema (DME) (4). DME is characterized by retinal thickening and swelling of the macular area and affects more than a quarter of patients with diabetes (5). Currently, the most common intervention for DME is focal and grid laser treatment, yet this approach may lead to retinal damage and a reduced visual field (6). Several factors that drive BRB breakdown in DR, including inflammation (7) and elevated vitreous levels of VEGF (8-10), have been suggested. This has led to the clinical introduction of corticosteroids and anti-

Conflict of interest: The authors have declared that no conflict of interest exists. Submitted: September 24, 2015; Accepted: May 12, 2016.

Reference information: J Clin Invest. 2016;126(8):3006-3022. doi:10.1172/JCI84767.
VEGFs for intravitreal injection. Regrettably, the former is associated with an increased incidence of cataracts and a harmful rise in intraocular pressure (11), while long-term intravitreal use of anti-VEGFs may lead to neuronal toxicity $(12,13)$ and potential geographic atrophy (14). New insights into the pathomechanisms that drive heightened permeability in DR may provide safer alternative therapeutic avenues.

While DR is primarily considered a microvascular disorder, there is accumulating evidence for early pathological events originating from the neuronal retina (15-19). Potential effector molecules with the propensity to mediate neurovascular communication in retinal health and disease are classical neuronal guidance cues and include netrins $(20,21)$, semaphorins (17, 18, 22-24), ephrins $(25,26)$, and slits (27-29). While initially identified as mediators of neuronal circuit formation during embryogenesis, these cues play equally prominent roles in vascular (30) and immune function $(18,31,32)$.

We have previously shown that netrin-1 produced by retinal neurons activates a program of reparative angiogenesis within microglia and initially restores functional vasculature to ischemic tissue during retinopathy (21). Netrin-1 is a laminin-related protein $(33,34)$ that exerts dichotomous biological effects, depending on the receptor it binds (35) and the intracellular cAMP levels (36). Canonically, signaling through deleted-in-colorectal-cancer (DCC) leads to attraction (37), while signaling through uncoordinated 5B (UNC5B) mediates repulsion or growth inhibition (38). 
Table 1. Characteristics of patients

\begin{tabular}{|c|c|c|c|c|c|c|c|c|c|}
\hline \multicolumn{5}{|c|}{ Control patients } & \multicolumn{5}{|c|}{ Diabetic patients } \\
\hline Pathology & OCT & Mean \pm SEM & Age & Mean \pm SEM & Pathology & OCT & Mean \pm SEM & Age & Mean \pm SEM \\
\hline ERM & $<250$ & $<250$ & 54 & $67 \pm 4.98$ & $\mathrm{DME}^{\mathrm{A}}$ & 505 & $444.4 \pm 39.05$ & 61 & $74 \pm 3.40$ \\
\hline ERM & $<250$ & & 72 & & $\mathrm{DME}^{\mathrm{A}}$ & 397 & & 75 & \\
\hline ERM & $<250$ & & 65 & & $\mathrm{DME}^{\mathrm{A}}$ & 559 & & 81 & \\
\hline & & & & & $\mathrm{DME}^{\mathrm{A}}$ & 421 & & 77 & \\
\hline
\end{tabular}

AType 2 diabetes.

Here, we report that cleavage and fragmentation of netrin-1 by matrix metalloprotease 9 (MMP-9), and probably other collagenases, directly impacts barrier function in the diabetic retina. Specifically, a fragment corresponding to the VI (N-terminus) and $\mathrm{V}$ domains of full-length netrin-1 is elevated in the vitreous fluid of patients suffering from DME and in mouse retinae during experimental type 1 diabetes. The expression dynamics of MMP-9 mirror that of the VI-V fragment, and inhibition of MMP-9 reduces processing of full-length netrin-1. In contrast to full-length netrin-1, the VI-V fragment has the capability to provoke vascular permeability via the UNC5B receptor. Together, these findings suggest that cleavage of netrin- 1 may contribute to heightened vascular permeability in DR.

\section{Results}

Truncated netrin-1 isoforms are present in the vitreous humor of patients with DME. To assess the potential involvement of netrin-1 in DR, we first investigated protein levels in vitreous humor from patients with DME, selected according to their macular thickness as determined by spectral-domain optical coherence tomography (SD-OCT) (>250 $\mu \mathrm{m})$ (Table 1). Representative SD-OCT 3D retinal maps and cross sections are shown in Figure 1, A and B. Full-length netrin-1 is a 604-aa protein comprising the N-terminal to the C-terminal of one laminin domain (domain VI), 3 laminin EGF-like domains (domains $\mathrm{V}_{1}, \mathrm{~V}_{2}$, and $\mathrm{V}_{3}$ ), and a netrin-like domain (NTR) module also called the $\mathrm{C}$ domain (Figure $1 \mathrm{C}$ ). We used a netrin- $1 \mathrm{Ab}$ with specificity to sequences within domain $\mathrm{V}$ for Western blot analysis (Figure 1C). Western blot analysis of equal volumes of vitreous (Figure 1D) revealed a major band at $72 \mathrm{kDa}$ corresponding to full-length netrin-1 (Figure 1D) and an increase in a $55-\mathrm{kDa}$ fragment of netrin-1 in patients with DME. According to their mass and prospective fragmentation profiles, netrin-1 bands at $55 \mathrm{kDa}$ were consistent with the proteolytic processing of full-length netrin-1 to remove one of its domains (Figure 1E). Densitometric analysis revealed the 55-kDa truncated form of netrin-1 to be significantly increased by approximately 8 -fold in patients with DME (healthy patients $1.000 \pm 0.053$, DME patients $8.433 \pm 3.038, P=0.0270$ ) (Figure $1 F$ ), coincident with a nonsignificant drop in the amount of full-length netrin-1 (healthy patients $1.000 \pm 0.2474$, DME patients $0.443 \pm 0.1476$ ) (Figure $1 G$ ). Importantly, higher levels of the truncated $55-\mathrm{kDa}$ form of netrin-1 correlated with increased retinal edema as determined by SD-OCT $\left(r^{2}=0.5235, P<0.05\right)$ (Figure $1 \mathrm{H}$ ), whereas we were unable to establish a significant correlation between the drop in full-length netrin-1 levels and increased retinal edema $\left(r^{2}=0.2283\right)$ (Figure 1I). Together, these data suggest that patients suffering from DME show elevated vitreous levels of a $55-\mathrm{kDa}$ truncated form of netrin-1.

Truncated netrin-1 is highly expressed in retinae during streptozotocin-induced diabetes. Given the induction of truncated fragments of netrin-1 in the vitreous of patients with DME, we next investigated the kinetics of generation of netrin- 1 fragments in a streptozotocin (STZ) mouse model of type 1 diabetes. STZ was administered to 6-week-old C57BL/6J mice over 5 consecutive days (Figure 2A), and the mice were considered diabetic when their glycemic levels exceeded $17 \mathrm{mM}(300 \mathrm{mg} / \mathrm{dl}$ ) (Supplemental Figure 1A). Eight weeks after STZ treatment, retinal vascular permeability rose by more than 2-fold when compared with that of control mice injected with vehicle (Supplemental Figure 1B), and FITC-dextran perfusion corroborated the presence of leaky vessels (Supplemental Figure 1C). Physiological parameters of the STZ-treated mice are presented in a polygonal graph in Supplemental Figure 1D.

IHC and 3D reconstruction of retinal cross sections from healthy adult mice revealed that netrin-1 was largely confined to the ganglion cell layer (GCL) (Figure 2B). Expression was quantitatively confirmed by quantitative PCR (qPCR) after laser capture microdissection (LCM) of the GCL, inner nuclear (INL), and outer nuclear layer (ONL) (GCL: $1.000 \pm 0.0338$, INL: $0.0258 \pm 0.1236$, ONL: $0.0635 \pm 0.0006, P<0.0001$ ) (Figure $2 \mathrm{C}$ ).

We subsequently assessed retinal expression of netrin-1 protein by Western blotting 4, 6, and 8 weeks after STZ treatment (Figure 2D). Netrin-1 protein levels were significantly increased in STZ-treated mice when compared with levels in citrate-treated control mice ( 4 weeks: $1.000 \pm 0.032$ [citrate], $1.151 \pm 0.059$ [STZ], NS, $n=7 ; 6$ weeks: $1.000 \pm 0.2394$ [citrate], $1.944 \pm 0.178$ [STZ], $P<0.05, n=3$; and 8 weeks: $1.000 \pm 0.064$ [citrate], $1.872 \pm 0.222$ [STZ], $P<0.01, n=7$ ) (Figure 2E). In accordance with our finding in diabetic human vitreous (Figure 1D), we observed a significant induction of a 55-kDa netrin-1 fragment (4 weeks: $1.000 \pm 0.045$ [citrate], $1.369 \pm 0.089$ [STZ], $P<0.01 ; 6$ weeks: $1.000 \pm 0.031$ [citrate], $2.243 \pm 0.034$ [STZ], $P<0.001$; and 8 weeks: $1.000 \pm$ 0.081 [citrate], $1.899 \pm 0.1727$ [STZ], $P<0.001$ ) (Figure $2 \mathrm{~F}$ ). The discrepancy between netrin-1 levels, which are reduced in the vitreous of DME patients and elevated in mouse diabetic retinae, may be related to the stage of the disease.

We then purified netrin- 1 by IP using an Ab specifically targeting the N-terminal extremity of netrin-1 (clone NORA-1 ALX-804838-C100) (Figure 2G). Gel electrophoresis of the resulting immu- 
A

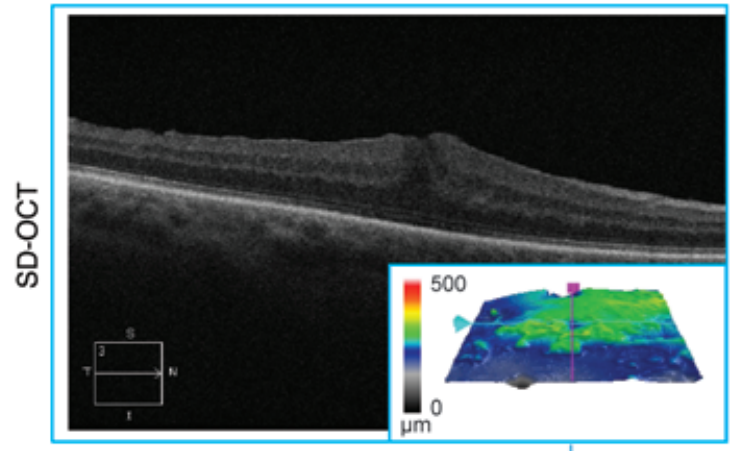

B

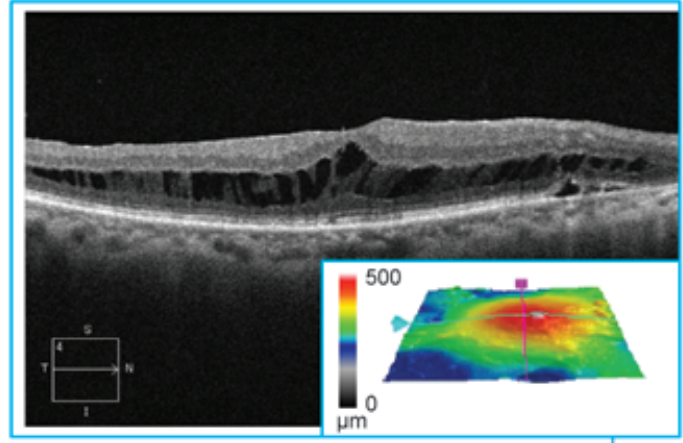

C

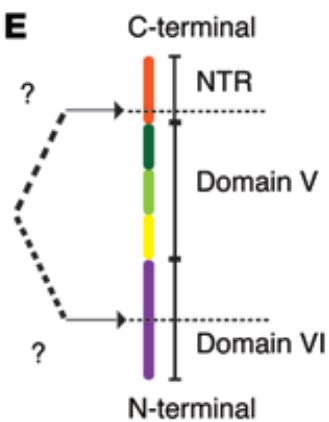

E

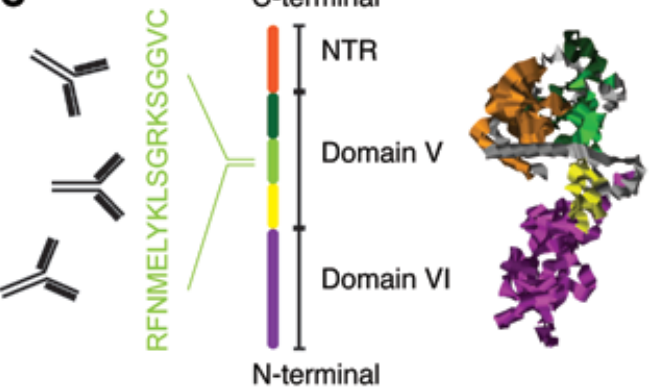

$\mathbf{F}$

D
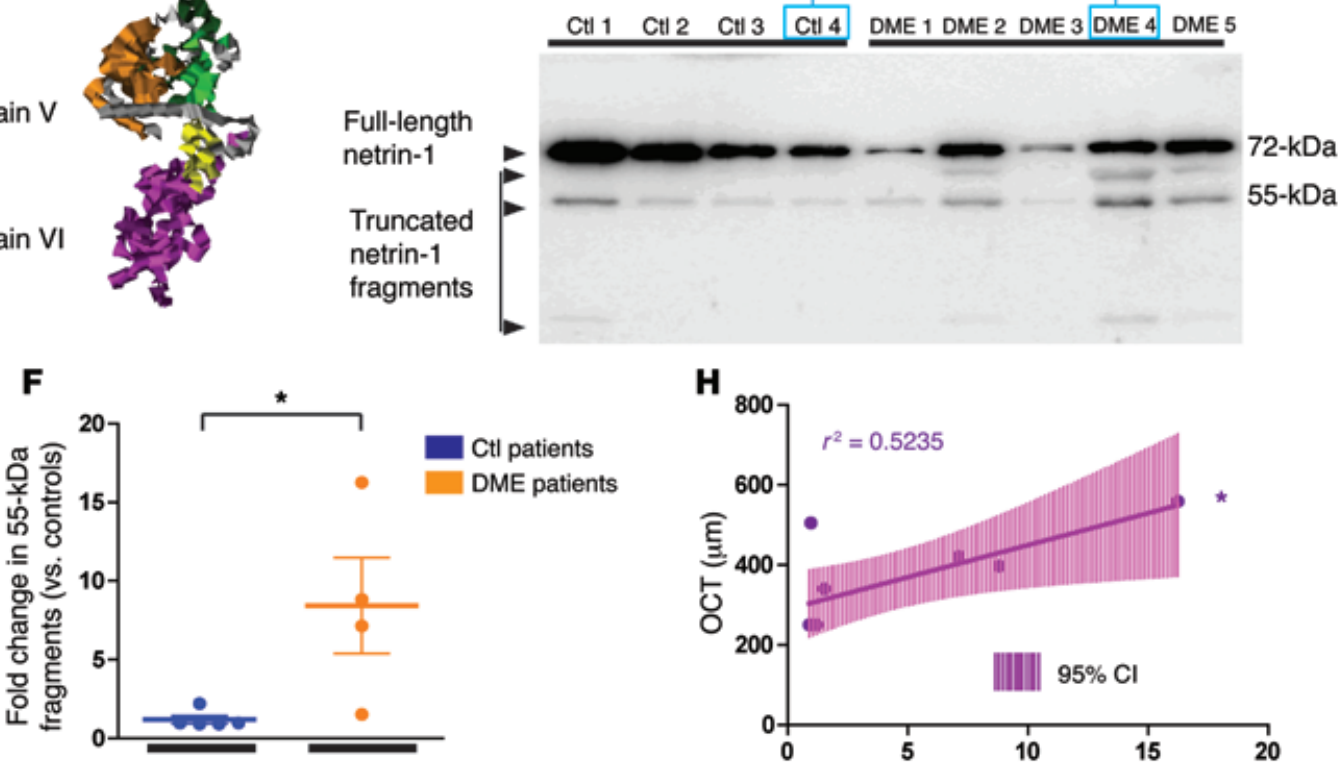

Ctl patients

DME patients

\section{H}
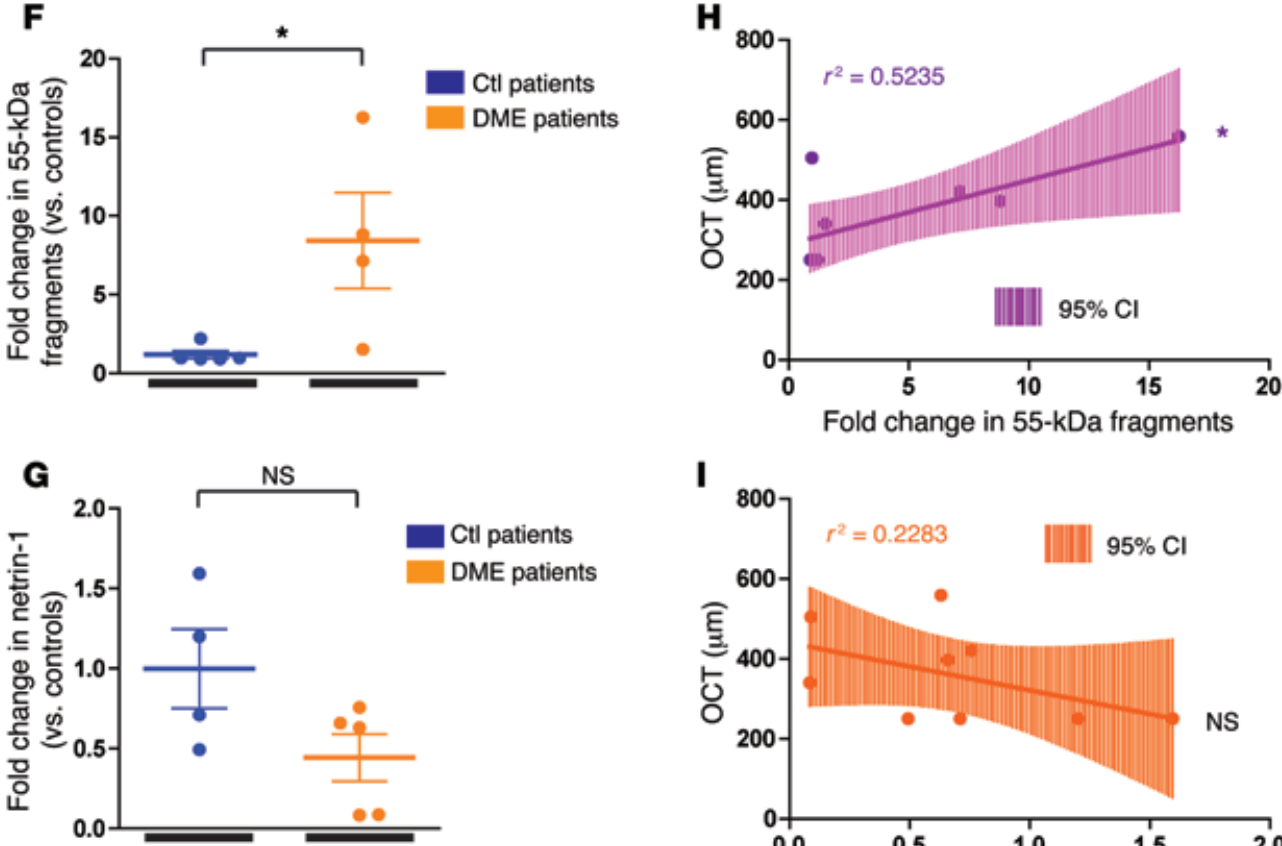

I

CIl patients

DME patients

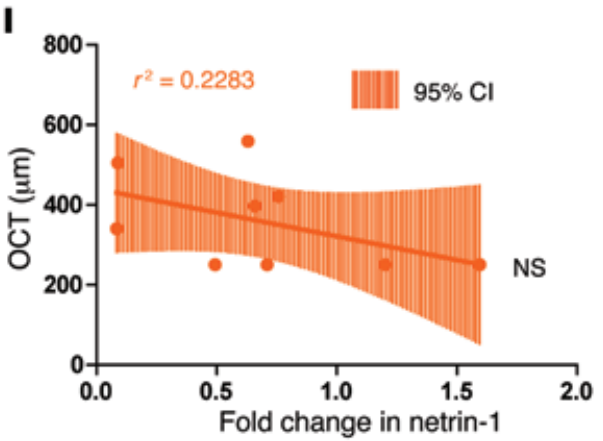

Figure 1. Elevated levels of truncated netrin-1 in the vitreous humor of patients with DME. (A and B) 3D retinal maps and SD-OCT of control eye (left) versus eyes of patients with DME (right). The severity of retinal swelling was particularly evident in the central foveal zones. (C) Netrin-1 Ab targeting a specific peptide sequence in netrin-1 domain $V(60)$. (D) Western blot analysis of equal volumes of vitreous humor revealed the presence of full-length netrin-1 in all samples and of an approximately 55-kDa netrin-1 fragment in a number of patients with DME $(n=4-5)$. (E) Schematic diagram of a 55-kDa netrin-1 fragment. (F) Quantification of signal density revealed an approximately 8-fold increase in the VI-V fragment in vitreous humor $(n=4-5)$. (C) Quantification of signal density revealed an approximately $50 \%$ decrease in full-length netrin-1 levels ( $n=4-5$, NS). (H) Correlation curve between the $55-\mathrm{kDa}$ fragment and OCTs, showing that $52 \%$ of the OCT variation was associated with the $55-\mathrm{kDa}$ fragment variation. (I) Correlation curve revealed no significant correlation between full-length netrin-1 and OCTs. Data are expressed as the mean \pm SEM. ${ }^{*} P<0.05$, by 2 -tailed Student's $t$ test. Ctl, control. 


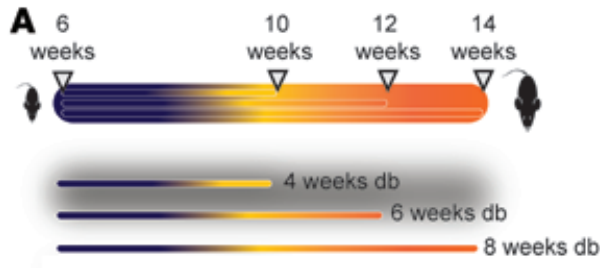

B
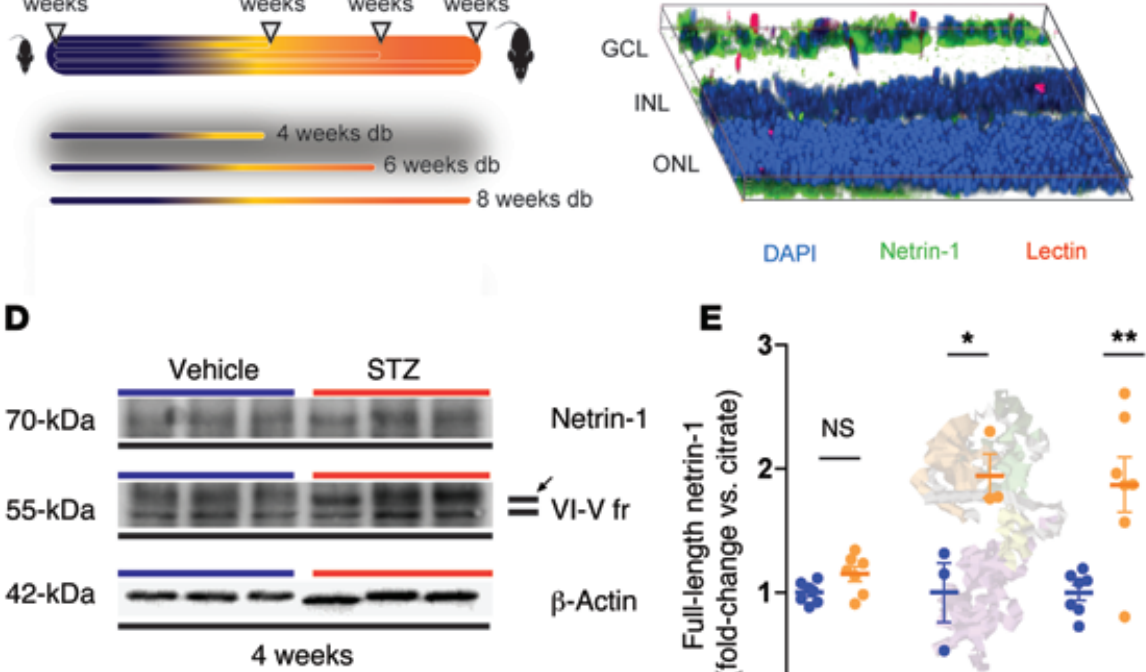

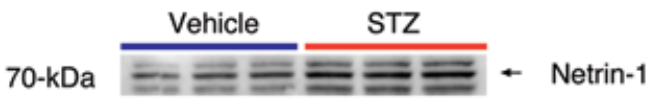

55-kDa

42-kDa

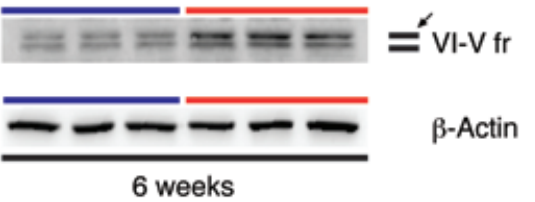

$70-\mathrm{kDa}$

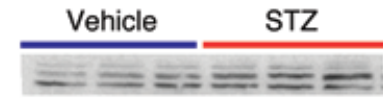

$55-\mathrm{kDa}$

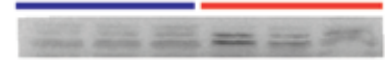

42-kDa

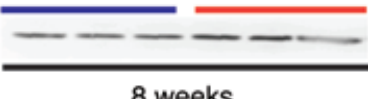

$\leftarrow$ Netrin-1

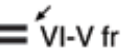

$\beta$-Actin
$\mathbf{E}$

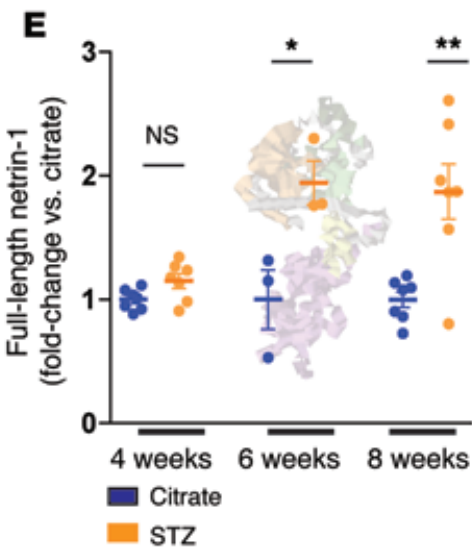

$\mathbf{F}$

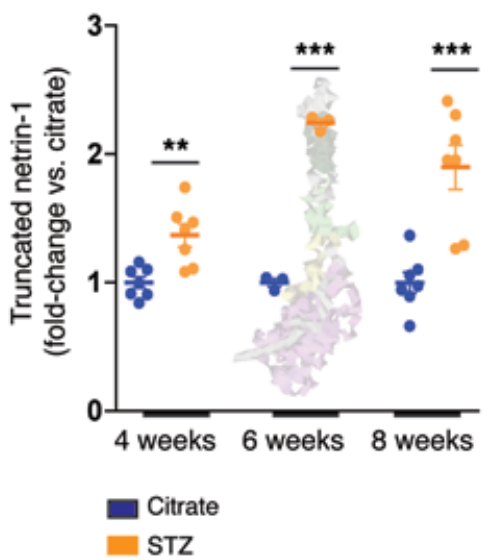

C

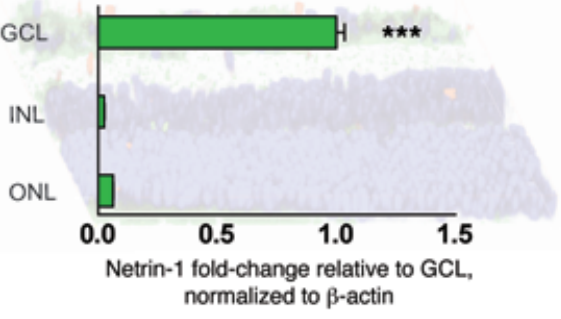

G

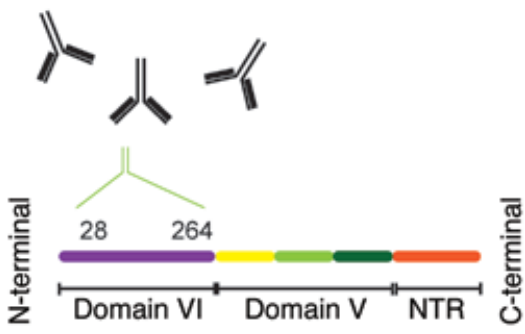

H

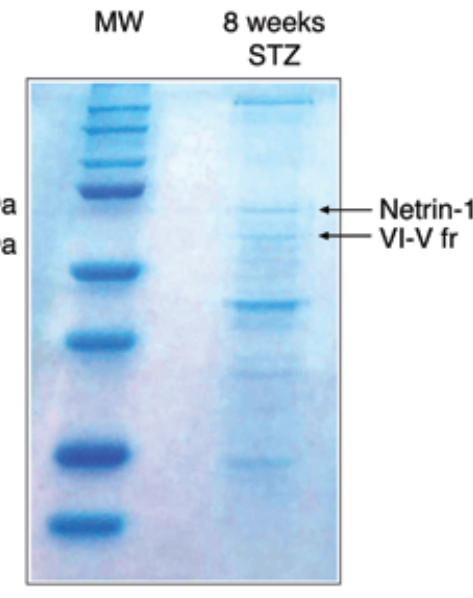

IP: Netrin-1

Figure 2. Truncated netrin-1 is highly expressed in early phases of diabetes in a mouse STZ model. (A) STZ or citrate vehicle was administered to 6 -weekold C57BL/6] mice. Retinae were then extracted at 4, 6, and 8 weeks of diabetes (db). (B) Netrin-1 localization in the GCL in adult murine retina. Representative confocal images of isolectin B4- (vessel stain), DAPI- (nuclei stain), and netrin-1-immunostained retinal cross sections (20- $\mu \mathrm{m})$, reconstructed in 3D $(n=3)$. (C) LCM of the GCL, INL, and ONL, followed by qPCR confirmed expression of netrin-1 in the GCL $(n=3)$. (D) Representative Western blot analyses of retinae confirmed increased levels of the VI-V fragment at 4,6 , and 8 weeks of diabetes $(n=3)$. VI-V fragment corresponds to the upper band indicated by the arrow. (E) Quantification of full-length netrin-1 signal density at $4(n=7), 6(n=3)$, and 8 weeks $(n=7)$. (F) Quantification of VI-V fragment signal density at $4(n=7), 6(n=3)$, and 8 weeks $(n=7)$. (C) Netrin-1 Ab targeting the aa sequence of domain VI of netrin-1 (clone NORA-1). (H) Gel electrophoresis of immunoprecipitated netrin-1 ( $n=10$ diabetic retinae). Data are expressed as the mean \pm SEM. ${ }^{*} P<0.05,{ }^{* *} P<0.01$, and ${ }^{* * *} P<0.001$, by 2 -tailed Student's $t$ test (E and $\mathbf{F}$ ) and 1-way ANOVA with Tukey's post-hoc test (C). fr, fragment.

noprecipitate was stained with SimplyBlue SafeStain, and the results revealed a banding pattern similar to that shown in Figure 1D (Figure $2 \mathrm{H}$ ), demonstrating that the $55-\mathrm{kDa}$ murine truncated netrin- 1 contains domain VI - and likely corresponds to netrin-1 devoid of the netrin-like domain (NTR) module - or the VI-V fragment.

The VI-V fragment of netrin-1 plays a role in early breakdown of the BRB. Given the early induction of the VI-V fragment of netrin-1 in diabetic retinae (Figure 2), we sought to determine whether this protein contributes to the pathogenesis of DR. The time point at which the VI-V fragment of netrin-1 appears in the retina (Fig- ure 2D) is associated with heightened vascular permeability. We therefore generated a Myc-tagged recombinant form of the VI-V fragment and assessed its ability to mediate vascular permeability using 3 distinct models. First, we tested the ability of the VI-V fragment to induce retinal vascular permeability (Figure 3A). Intravitreal injection of the VI-V fragment significantly induced up to 2-fold more Evans blue (EB) extravasation when compared with vehicle control $(1.000 \pm 0.1112$ [vehicle], $1.891 \pm 0.1951$ [VI-V fragment, $3 \mathrm{nM}$ ], $P<0.01 ; 1.297 \pm 0.2423$ [full-length netrin- $1,3 \mathrm{nM}$ ], NS; $2.961 \pm 0.1151$ [VEGF, 2 nM],$P<0.001$ (Figure 3, A and B). 
A

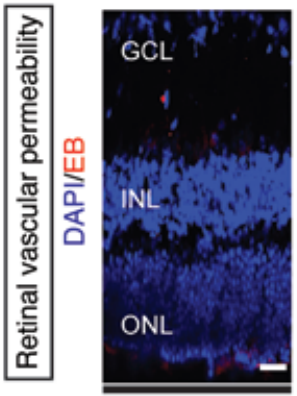

c

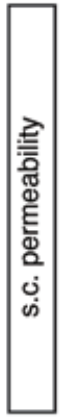

E

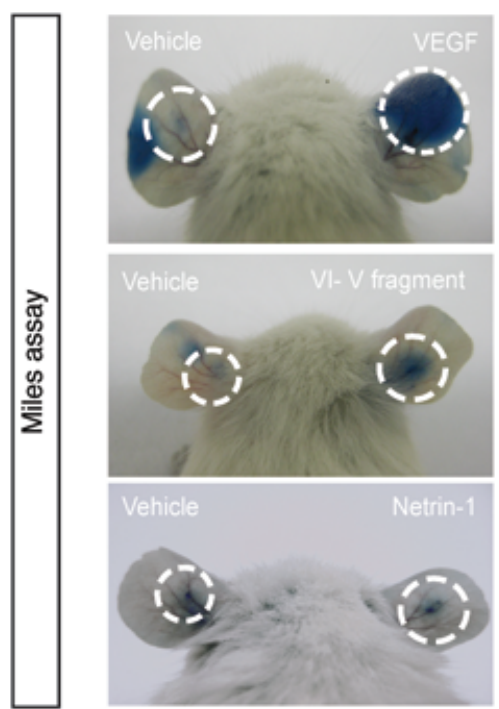

Vehicle

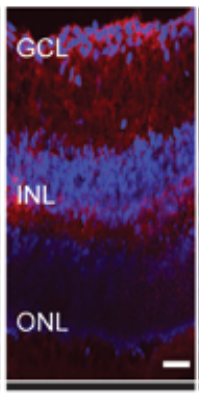

$\mathrm{VII} \mathrm{V} f \mathrm{r}$

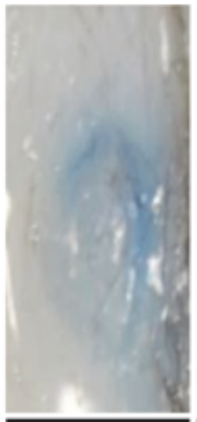

Vehicle

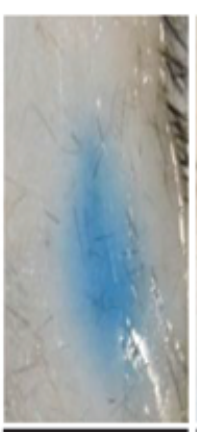

$\mathrm{VI}-\mathrm{V} \mathrm{fr}$

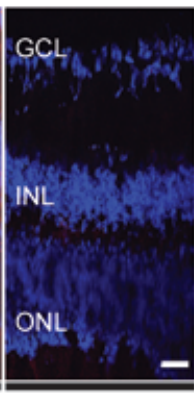

Netrin-1

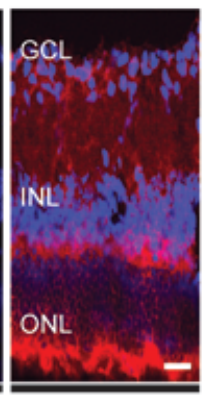

VEGF

\section{B}
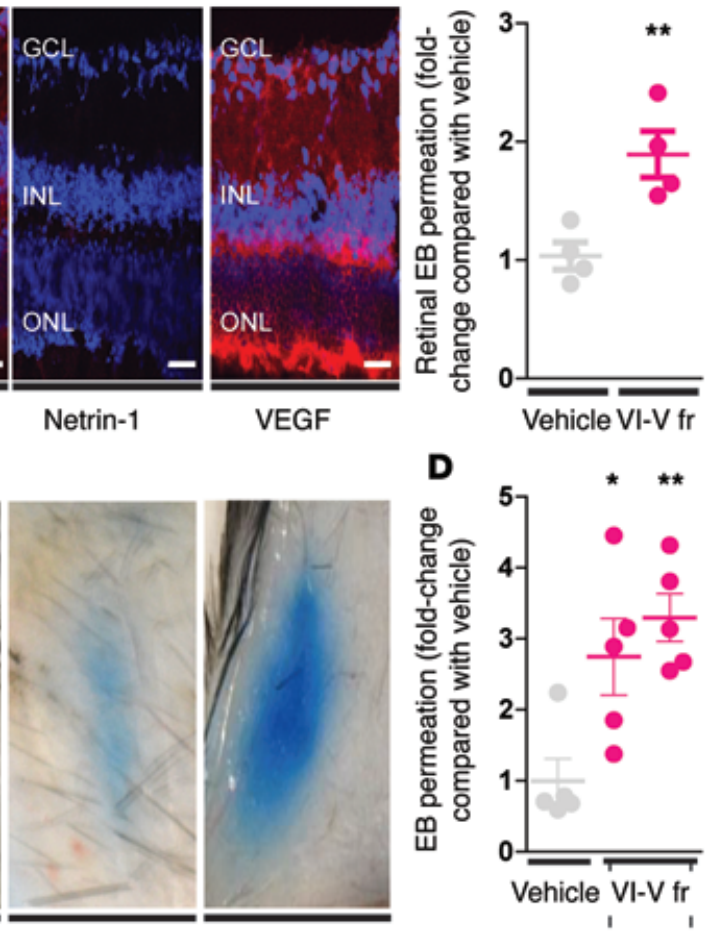

Netrin-1

VEGF
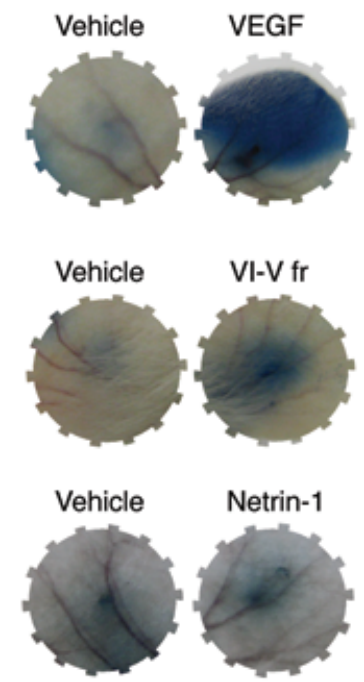

D

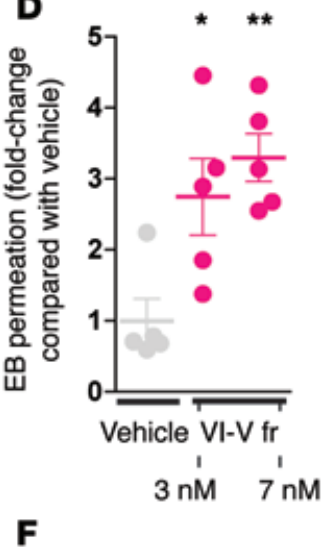

$\mathbf{F}$
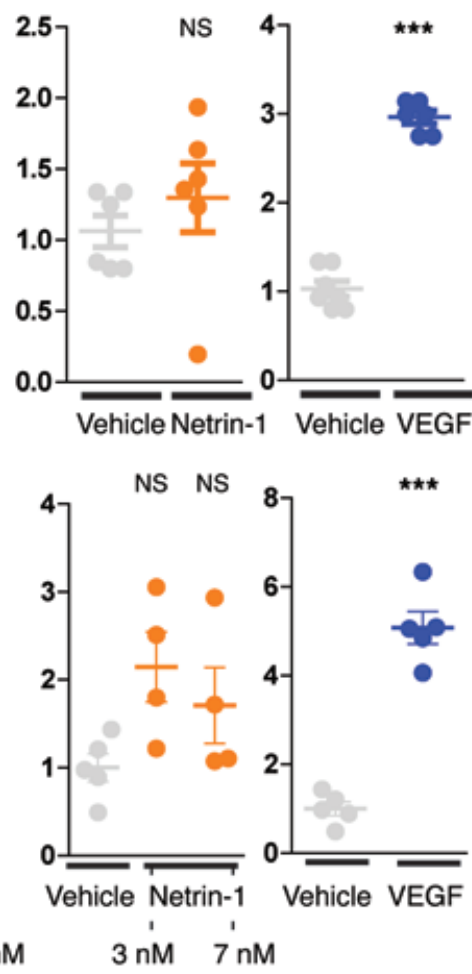
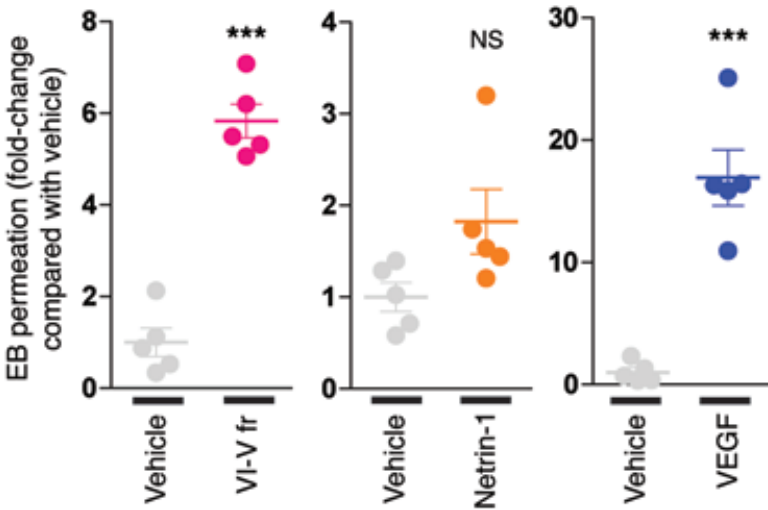

Figure 3. The VI-V fragment plays a role in early BRB breakdown. (A) Representative images of vascular leakage induced with vehicle (PBS), recombinant VI-V fragment (3 nM), full-length netrin-1 (3 nM), or recombinant VEGF (2 $\mathrm{nM})$ injected into the vitreous. Intravitreal injection resulted in a robust increase of retinal vasopermeability in eyes treated with the VI-V fragment of netrin-1 and with VEGF. $n=3$. Scale bars: $30 \mu \mathrm{m}$. (B) EB extravasation (vascular leakage) induced by VI-V fragment, full-length netrin-1, and recombinant VEGF, measured by overnight extraction of the dye and spectrophotometric quantification $(n=4-6)$. (C) Representative images of the vascular leakage induced with vehicle, synthetic VI-V fragment, full-length netrin-1, or recombinant VEGF s.c. injected into adult C57BL/6] mice $(n=5)$. Original magnification, $\times 2.5$. (D) EB extravasation (vascular leakage) induced by VI-V fragment, full-length netrin-1, and recombinant VEGF was assessed by overnight extraction of the blue dye in formamide and spectrophotometric quantification $(n=5)$. (E) Representative images of the vascular leakage induced with vehicle (PBS), recombinant VEGF ( $2 \mathrm{nM})$, full-length netrin-1 ( $3 \mathrm{nM})$, or recombinant $\mathrm{VI}-\mathrm{V}$ fragment $(3 \mathrm{nM})$ intradermally injected into CD-1 mouse ears $(n=5)$. Original magnification, $\times 1.6$. (F) Quantification of EB extravasation (vascular leakage) induced by VI-V fragment, full-length netrin-1, and recombinant VEGF $(n=5)$. Data are expressed as the mean \pm SEM. ${ }^{*} P<0.05,{ }^{*} P<0.01$, and ${ }^{* * *} P<0.001$, by 2-tailed Student's $t$ test (B and $\mathbf{F}$ ) and 1-way ANOVA with Tukey's post-hoc test (D). 
A
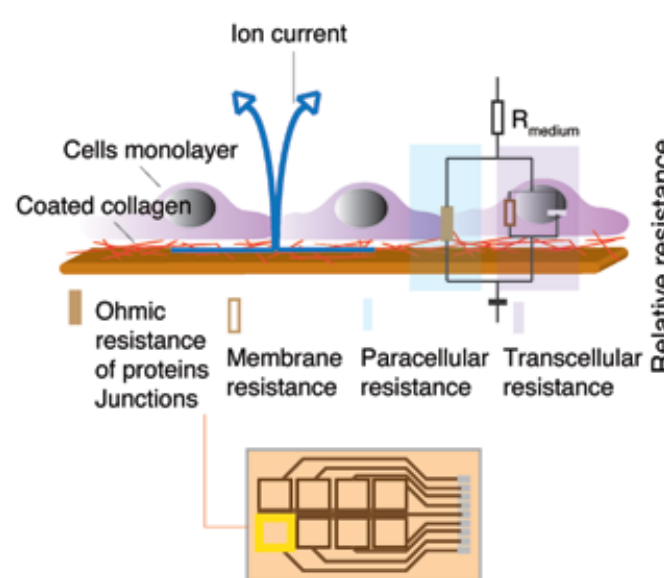

C

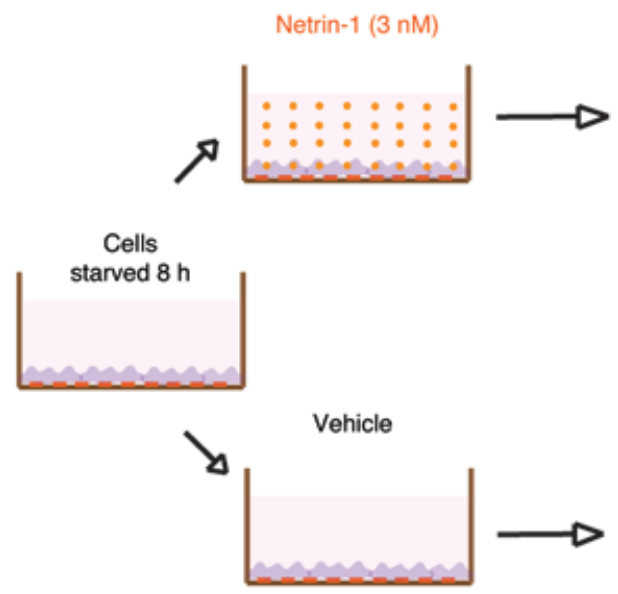

D
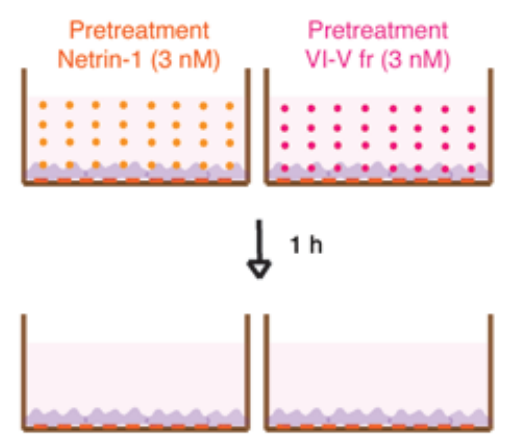

VEGF $(2 \mathrm{nM})$

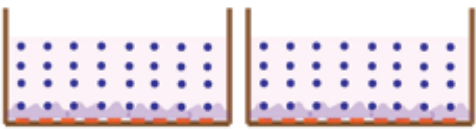

B
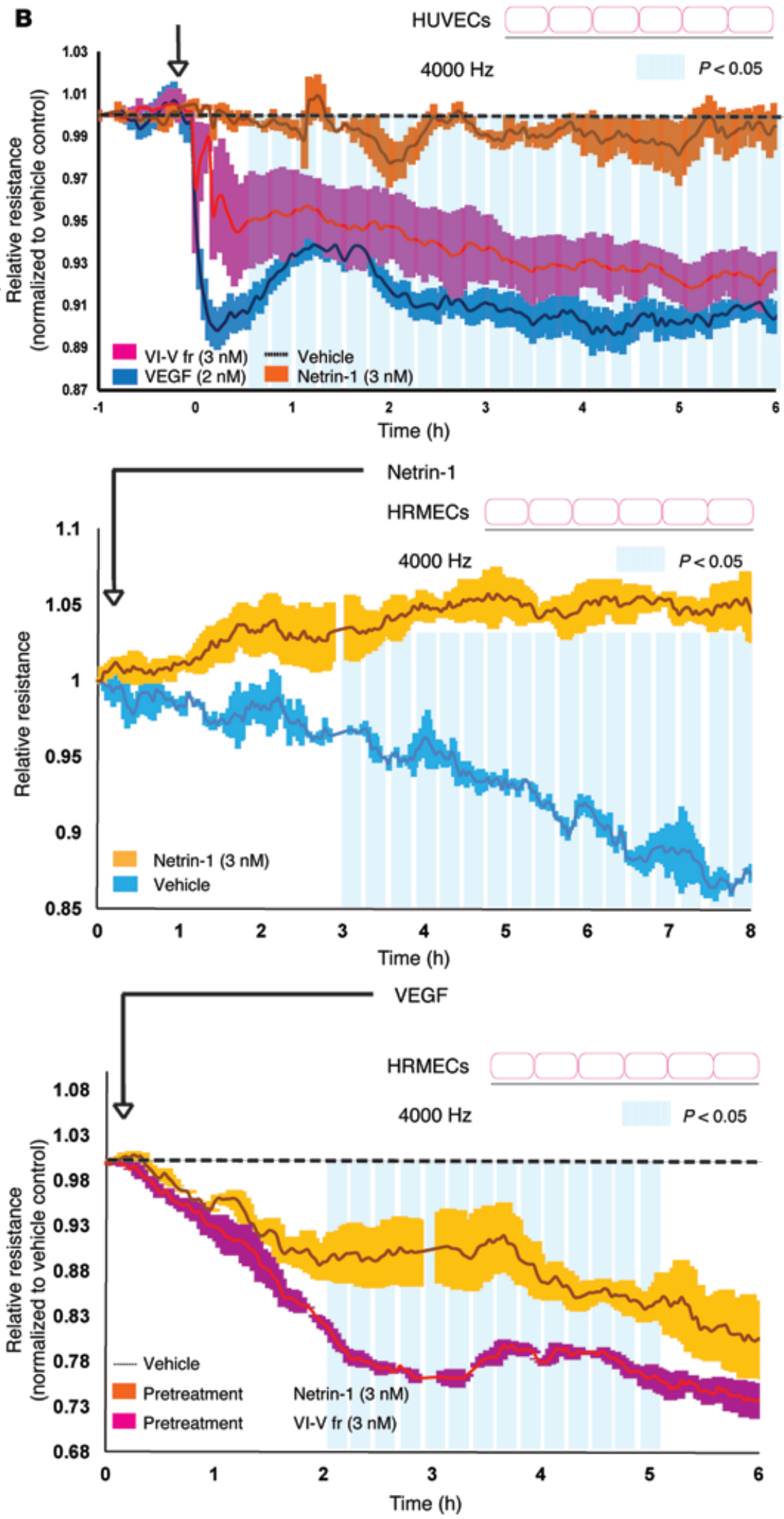

Figure 4. Netrin-1 preserves endothelial integrity, whereas the VI-V fragment disrupts endothelial barrier function. (A) ECIS measured live barrier function by assessing the resistance of the paracellular pathway between endothelial cells. $R_{\text {medium }}$, resistance of culture medium. (B) Paracellular resistance measured in real time by ECIS demonstrated that the VI-V fragment $(3 \mathrm{nM})$ compromised endothelial barrier function ( $1-6$ hours, $n=4)$, whereas netrin-1 ( $3 \mathrm{nM}$ ) preserved endothelial integrity with HUVECs. (C) Paracellular resistance measured in real-time by ECIS demonstrated that full-length netrin-1 delayed starving-induced (8 hours) monolayer breakdown (3-8 hours, $n=4$ ). (D) An endothelial cell monolayer pretreated with netrin-1 and exposed to VEGF was significantly more resistant than were endothelial cells pretreated with the VI-V fragment $(2-5$ hours, $n=4)$. Data are expressed as the mean \pm SEM. 
A

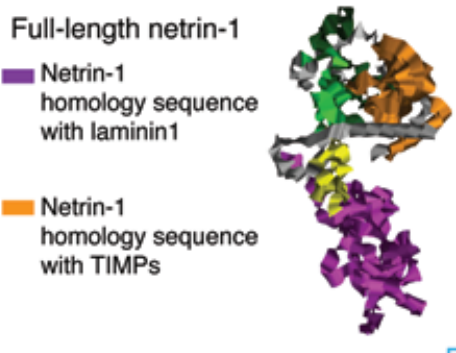

.

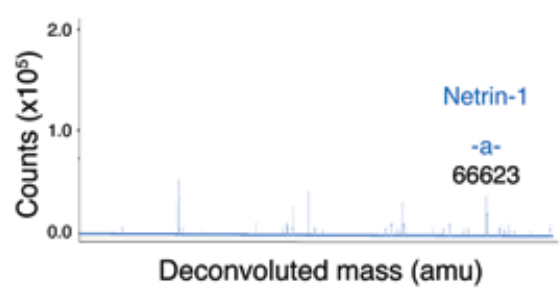

B

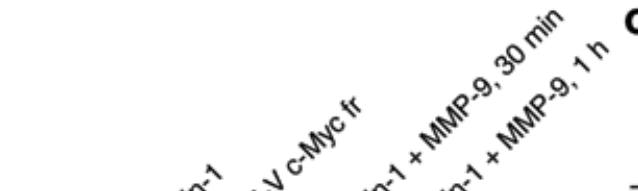

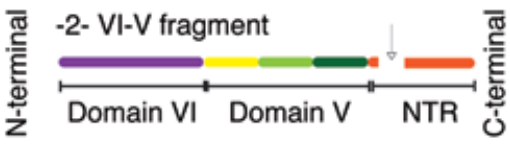

Figure 5. Generation of a VI-V fragment of netrin-1 by proteolytic cleavage in the diabetic retina. (A) Robetta 3D reconstruction of full-length netrin-1, highlighting netrin-1 homology with laminin (domain VI) and TIMPs (NTR module). (B) Representative Western blot analysis of full-length netrin-1 (3 nM) cleavage into the VI-V fragment by MMP-9 (c-Myc-tagged VI-V fragment was used as a control), with deconvoluted mass spectra showing a mass of netrin-1 standard, $66.62 \mathrm{kDa}$ (blue) and cleaved netrin-1 fragments of $55.9 \mathrm{kDa}$ and $34.51 \mathrm{kDa}(n=3$ ). rh, recombinant human. (C) Representative diagram highlighting the cleavage sites.

We next performed a modified Miles assay with s.c. injections of vehicle (PBS); the VI-V fragment ( 3 and $7.5 \mathrm{nM}$ ); full-length netrin-1 ( 3 and $7.5 \mathrm{nM}$ ); or recombinant VEGF (Figure $3 \mathrm{C}$ ). As above, the $\mathrm{VI}-\mathrm{V}$ fragment induced approximately 3 -fold more vascular leakage than was seen in vehicle controls $(1.000 \pm 0.3110$ [vehicle], $2.744 \pm 0.537$ [VI-V fragment, $3 \mathrm{nM}$ ], $P<0.05 ; 3.295 \pm 0.336$ [VI-V fragment, $7.5 \mathrm{nM}$ ], $P<0.01 ; 2.046 \pm 0.402$ [full-length netrin-1, 3 $\mathrm{nM}$ ], NS; $1.708 \pm 0.4346$ [full-length netrin-1, $7.5 \mathrm{nM}$ ], NS; $5.078 \pm$ 0.3658 [VEGF, $2 \mathrm{nM}$ ], $P<0.001$ ) (Figure 3, C and D). Finally, we performed an auricular Miles assay of s.c. permeability, whereby vehicle (PBS), the VI-V fragment (3 nM), and recombinant VEGF $(2 \mathrm{nM})$ were compared in auricular injections (Figure 3E). Quantification revealed that the VI-V fragment induced approximately 6-fold more permeability when compared with vehicle controls, yet less than was observed with VEGF $(1.000 \pm 0.3126$ [vehicle], $5.832 \pm 0.3658$ [VI-V fragment], $P<0.001 ; 1.824 \pm 0.3539$ [fulllength netrin-1], NS; $16.93 \pm 2.282$ [VEGF], $P<0.001$ ) (Figure 3F). In all paradigms tested, the VI-V fragment of netrin-1 significantly induced vascular permeability, albeit less potently than did VEGF.

The VI-V fragment of netrin-1 compromises endothelial barrier function. To corroborate the capacity of the VI-V fragment of netrin-1 to influence vascular permeability, we next measured variations in impedance on endothelial monolayers by electric cell-substrate impedance sensing (ECIS) (Figure 4A). Exposure of HUVEC s to the VI-V fragment of netrin-1 ( $3 \mathrm{nM})$ decreased impedance (normalized to vehicle), whereas treatment with netrin-1 (3 $\mathrm{nM})$ maintained monolayer integrity $(1-6$ hours, $P<0.05)$ (Figure $4 \mathrm{~B}$ ). As demonstrated in vivo (Figure 3 ), the VI-V fragment was less potent than VEGF. Conversely, upon starving cells for 8 hours, exposure of human retinal microvascular endothelial cells (HRMECs) to full-length netrin-1 delayed starvation-induced monolayer breakdown (Figure 4C), possibly due to the prosurvival effects of netrin- 1 (normalized to the resistance value at $t 0,3-8$ hours, $P<0.05)$. We next determined whether full-length netrin-1 or the VI-V fragment could affect VEGF-induced permeability. HRMEC monolayers pretreated with netrin- $1(3 \mathrm{nM})$ and exposed to VEGF $(2 \mathrm{nM})$ showed significantly less barrier function compromise than did those pretreated with the VI-V fragment (2-5 hours, $P<0.05$ ) (Figure 4D). Together with the above data, these findings highlight the divergent effect of full-length netrin-1 and the VI-V fragment with regard to endothelial barrier function.

MMP-9-mediated proteolysis generates fragments of netrin-1. Given the sequence homology of netrin-1 with tissue inhibitors of metalloproteinase (TIMPs) in its NTR module (ref. 39 and Figure $5 \mathrm{~A}$ ), we sought to determine whether netrin- 1 could be a substrate for collagenases. MMP-9 is a collagenase that is induced in DR and has documented roles in vascular permeability (40) (Figure 6A). We therefore incubated full-length netrin-1 with MMP- 9 at $37^{\circ} \mathrm{C}$ and monitored fragmentation at different time points (30 and 60 min) by Western blot analysis. At 30 minutes, a fragment corresponding to the predicted molecular weight of the VI-V peptide was detected, while netrin-1 itself was being degraded (Figure 5B). A c-Myc-tagged construct of the VI-V fragment was used as a control and hence accounts for the slight shift in bands between recombinant VI-V and MMP-9-generated VI-V. Importantly, the observed patterns of fragmentation are reminiscent of those seen in murine 

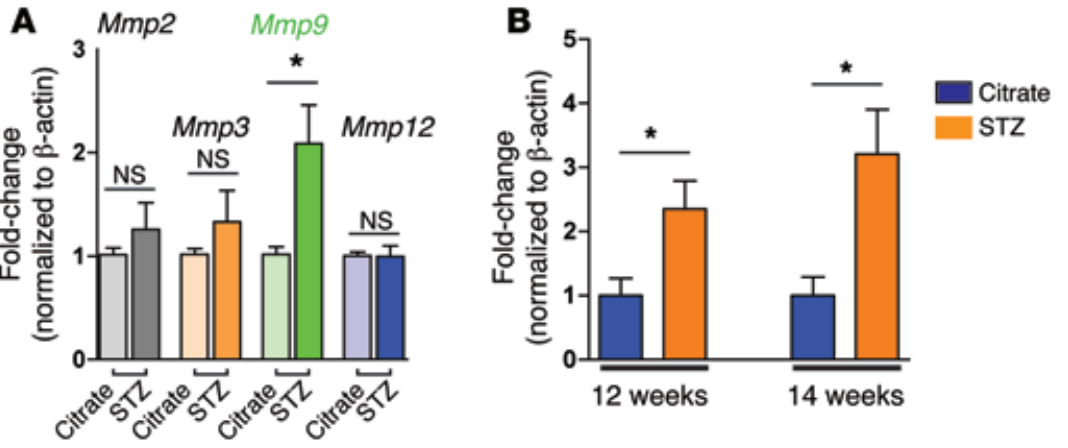

C

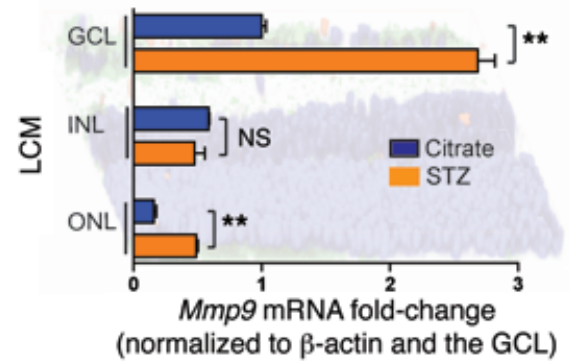

D

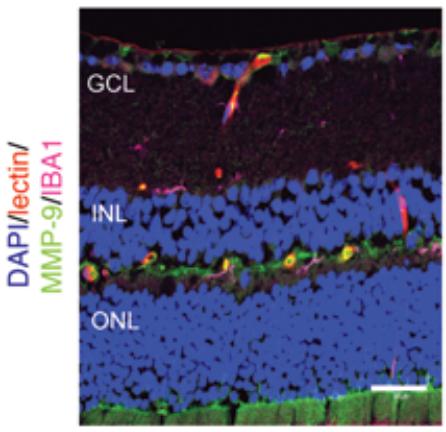

Citrate
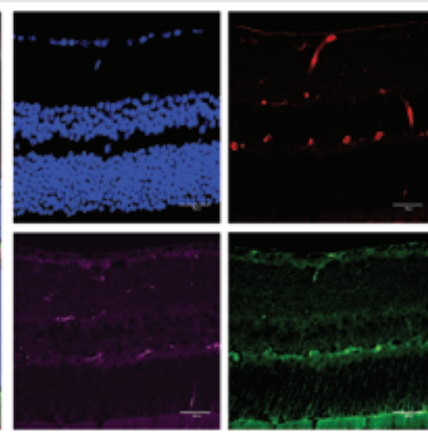

E

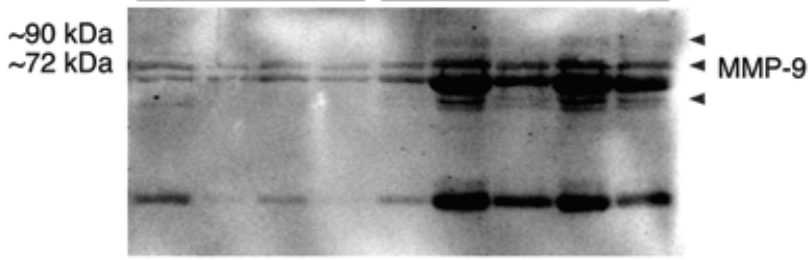

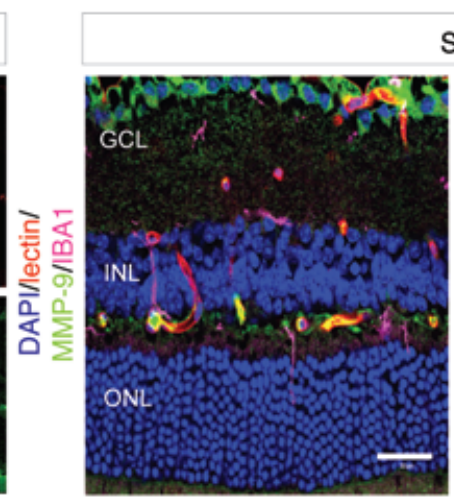

STZ
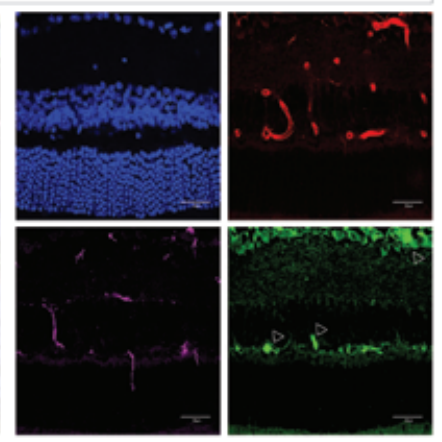

$\mathbf{F}$

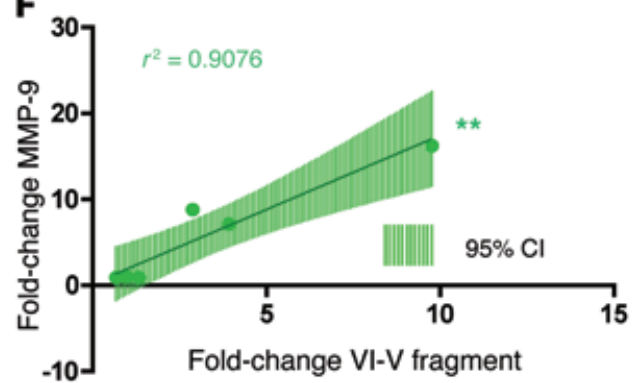

Figure 6. Increased levels of MMP-9 in the vitreous humor of patients with DME. (A) Murine retinal Mmp2, Mmp3, Mmp9, and Mmp12 mRNA levels at 8 weeks $(n=3)$. (B) Murine retinal Mmp9 mRNA was upregulated at 12 and 14 weeks of diabetes $(n=3)$. (C) LCM of the GCL, INL, and ONL, followed by qPCR of MMP-9 $(n=3)$. (D) Representative confocal images of isolectin B4- (vessels), IBA- (microglia), DAPI- (nuclei), and MMP-9-stained retinal cross sections at 8 weeks (citrate vs. STZ) revealed that murine retinal Mmp9 was mainly produced in the GCL $(n=4)$ ). Scale bars: $30 \mu \mathrm{m}$. (E) Western blot analysis of equal volumes of vitreous humor revealed the presence of MMP-9 in patients with DME $(n=4-5)$. (F) Correlation curve between MMP-9 and VI-V fragment generation showing that $90 \%$ of the VI-V fragment variation was associated with MMP-9 variation. Data are expressed as the mean \pm SEM. ${ }^{*} P<0.05$ and ${ }^{* *} P<0.01$, by 2-tailed Student's $t$ test.

diabetic retinal blots (Figure 2D). We used mass spectrometric analysis to determine the mass of full-length netrin-1 $(66.62 \mathrm{kDa})$ and the masses of 2 fragments resulting from the enzymatic cleavage of netrin-1 (Figure 5B). A first fragment with a mass of $55.99 \mathrm{kDa}$ corresponded to full-length netrin-1 with a cleaved NTR module, and a second one, with a mass of $34.52 \mathrm{kDa}$, corresponded to netrin-1 cleaved within the domain V (EGF-like 1) (Figure 5, B and C).

$M M P-9$ is induced in the vitreous humor of patients with DME. Collagenases are abundantly produced during development or during heightened inflammation. We therefore screened for transcripts of matricial collagenases ( $M m p 2, M m p 3, M m p 9$, and Mmp12) by qPCR at 8 weeks after induction of diabetes. We found $M m p 9$ to be the most robustly induced at this time point (Figure 6A), and it remained significantly elevated at 12 and 14 weeks of disease (12 weeks: $1.000 \pm 0.266$ [citrate], $2.348 \pm 0.440$ [STZ], $P<0.05 ; 14$ weeks: $1.000 \pm 0.290$ [citrate], $3.206 \pm 0.6947$ [STZ], $P<0.05$ )
(Figure 6B). LCM and real-time qPCR showed that Mmp9 increased by approximately 3-fold in diabetic GCLs and ONLs (GCL: 1.000 \pm 0.0338 [citrate], $2.688 \pm 0.1302$ [STZ], $P<0.01$; INL: $0.5864 \pm$ 0.0056 [citrate], $0.4742 \pm 0.0817$ [STZ], NS; ONL: $0.1574 \pm 0.0222$ [citrate], $0.4891 \pm 0.01413$ [STZ], $P<0.01$ ) (Figure 6C). Induction of MMP-9 was also corroborated by IHC in diabetic retinae at 8 weeks of diabetes, with elevated staining of MMP-9 in the GCL, in vessels, and partially in microglia (ionized calcium-binding adaptor molecule 1-positive [IBA1-positive] cells) (Figure 6D).

To assess the potential involvement of MMP-9 in DR, we next investigated its protein levels in vitreous from patients with DME. Western blot analysis of equal volumes of vitreous revealed that MMP-9 was increased in patients with DME (Figure 6E), corroborating data obtained in the STZ mouse model of diabetes (Figure 6 , A and B). Interestingly, we found that higher MMP-9 levels correlated with increased VI-V fragment levels $\left(r^{2}=0.9076, P<0.05\right)$ 
A

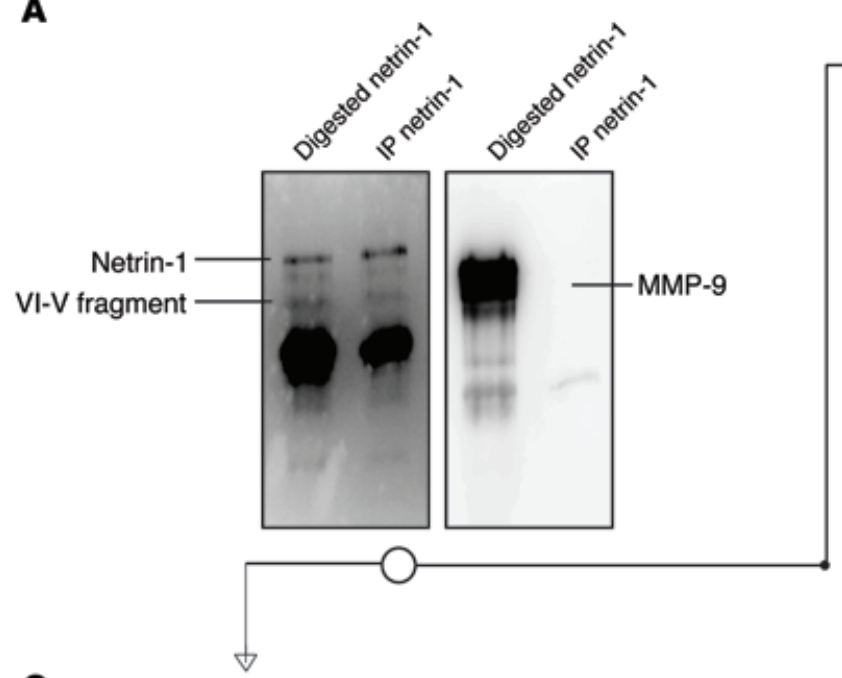

C
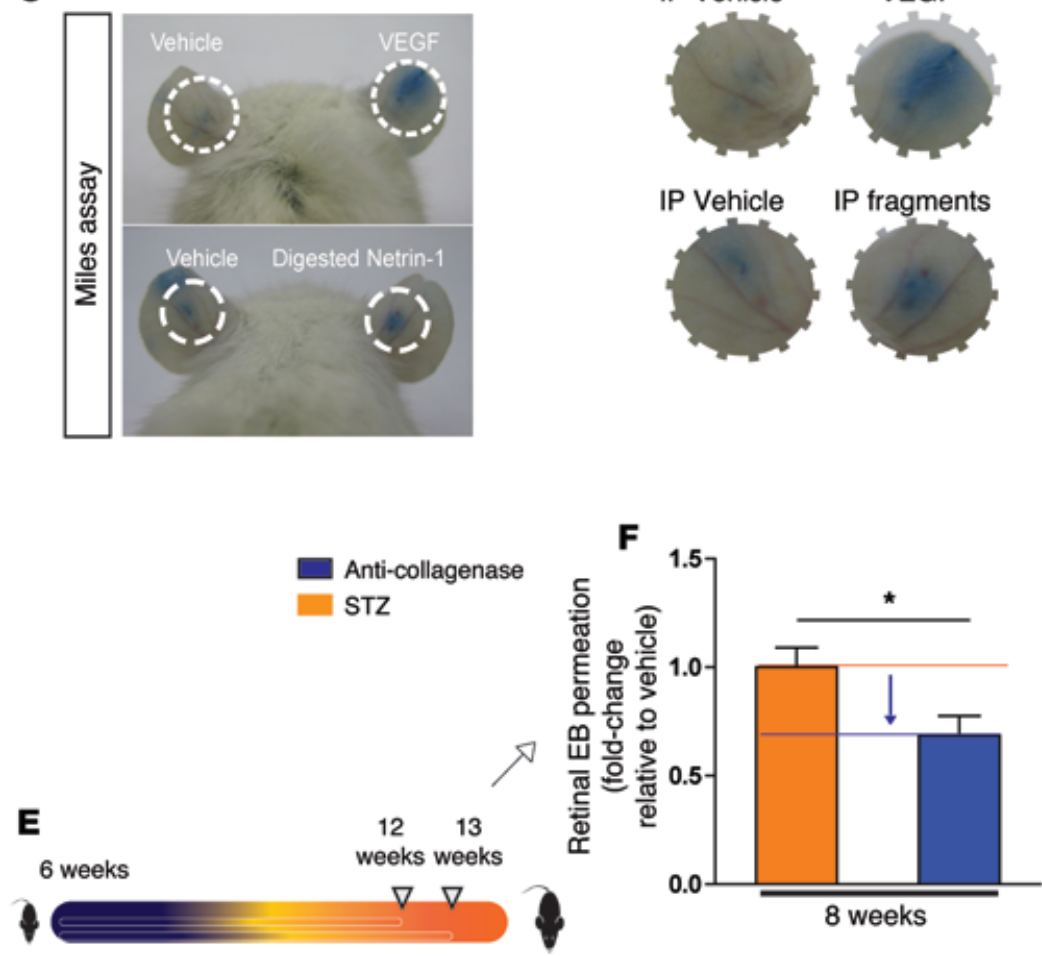

6 weeks diabetes

7 weeks diabetes
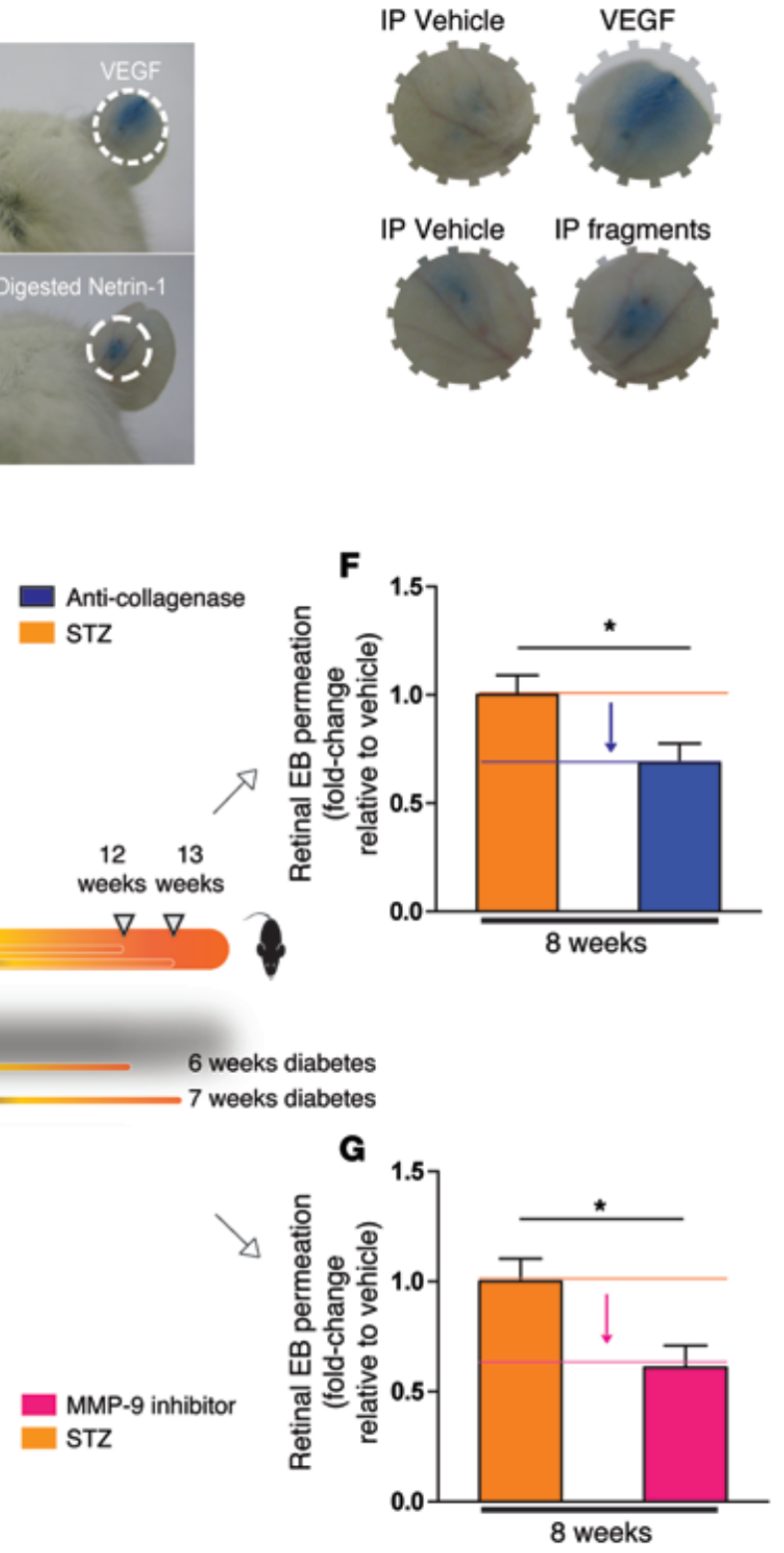

IP Vehicle IP fragments

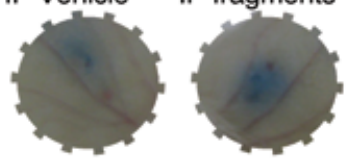

B HUVECS

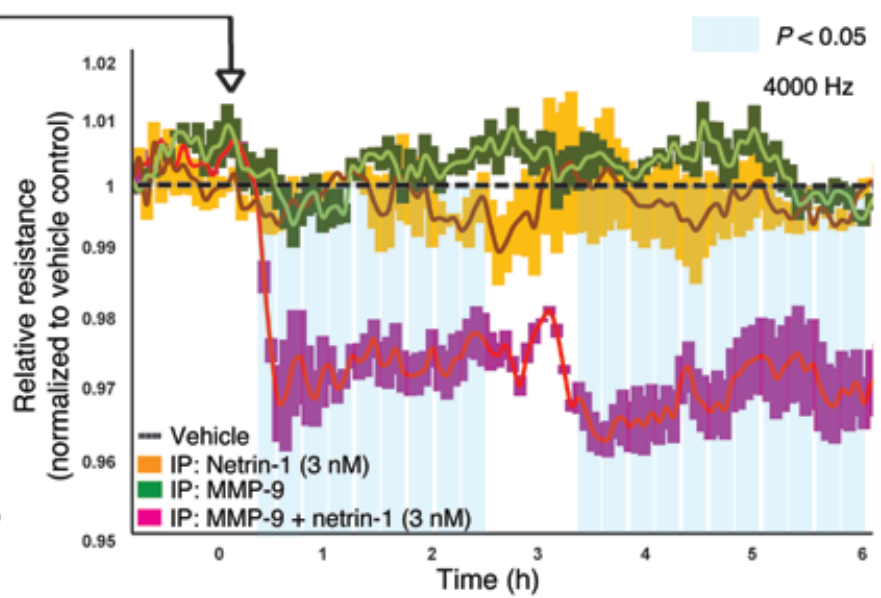

D

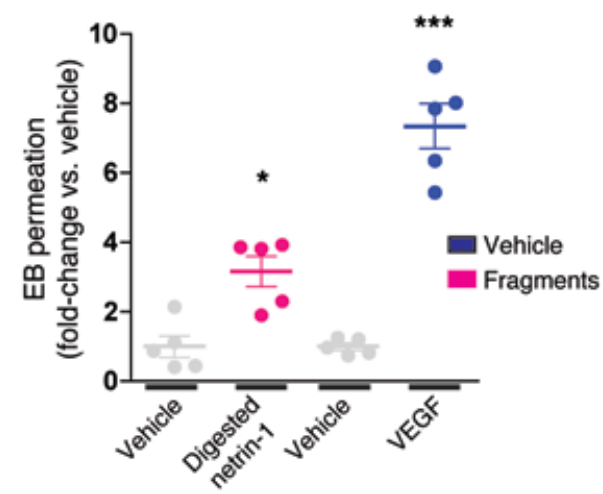

H

STZ STZ + MMP-9 inhibitor

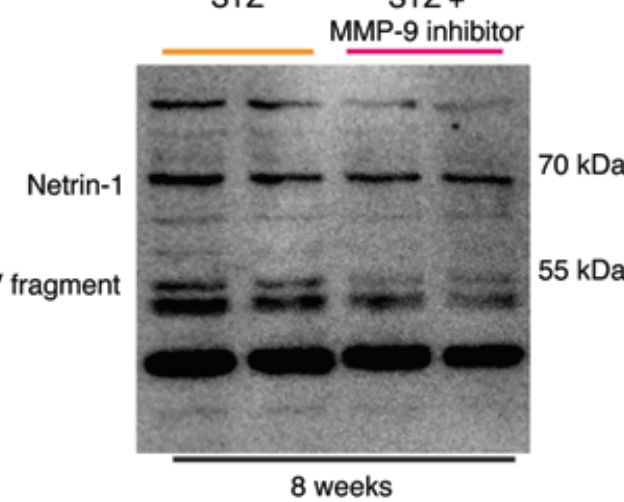

I

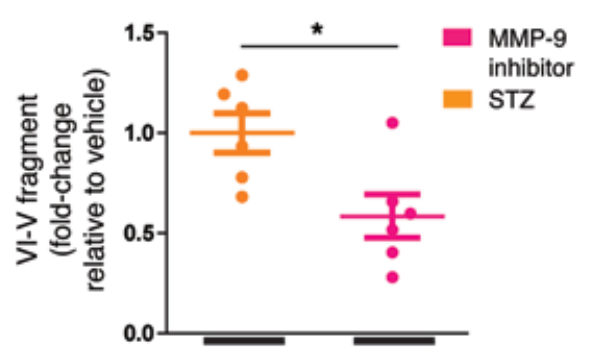


Figure 7. Inhibition of collagenases and MMP-9 in vivo reduces pathological vascular permeability. (A) Netrin-1 IP after MMP-9 digestion. Left panel: Representative Western blot of netrin-1; digested netrin-1 (MMP-9 plus netrin-1) versus netrin-1 fragments purified by IP. Right panel: Representative Western blot of MMP-9; digested netrin-1 (MMP-9 plus netrin-1) versus netrin- 1 fragments purified by IP $(n=3)$. (B) Paracellular resistance measured in real time by ECIS revealed that netrin-1 fragments reduced endothelial barrier function ( $0.3-6$ hours, $n=4)$, whereas netrin- 1 and MMP-9 controls did not change endothelial resistance. (C and D) Vascular leakage induced with vehicle, recombinant VEGF, or netrin-1 fragments purified by IP and injected into [57BL/6] mice ears and (D) quantification of EB extravasation (vascular leakage) induced by netrin-1 fragments, full-length netrin-1, and recombinant VEGF $(n=5)$. (E) Intravitreal injection of collagenase inhibitors into STZ mice at weeks 6 and 7 after induction of diabetes led to $(\mathbf{F})$ a $31 \%$ reduction in retinal permeability after 8 weeks of diabetes $(n=4)$. (C) Intravitreal injection of MMP-9 inhibitors into STZ mice at weeks 6 and 7 after induction of diabetes resulted in a 39\% reduction in retinal permeability after 8 weeks of diabetes $(n=4)$. ( $(H)$ Representative Western blot analysis showing that inhibition of MMP-9 decreased the concentration of VI-V fragments in diabetic retina and (I) quantification of the relative levels of VI-V fragments following MMP-9 inhibitor treatment in diabetic retina $(n=6)$. Data are expressed as the mean \pm SEM. ${ }^{*} P<0.05$ and ${ }^{* *} P<0.001$, by 1 -way ANOVA with Tukey's post-hoc test (D) or 2-tailed Student's $t$ test (F, G, and I).

(Figure 6F). Together, these data suggest that MMP-9 is upregulated in patients with DME and has the propensity to promote cleavage of netrin-1.

Inhibition of collagenases or MMP-9 in vivo reduces pathological vascular permeability. To further investigate whether cleavage of netrin-1 is driving vasopermeability, we examined the behavior of HUVEC monolayers stimulated with fragments produced in vitro by incubation of netrin-1 ( $3 \mathrm{nM})$ with MMP-9. Fragments were generated as described for Figure 5B, collected after 30 minutes, immunoprecipitated to remove MMP-9 (Figure 7A), and exposed to the endothelial monolayer by ECIS. IP of netrin-1 or MMP-9 alone served as a control (Figure 7A, right panel). A drop in HUVEC impedance following exposure to products of MMP-9-mediated degradation of netrin-1 suggests processing of netrin-1 into vasoactive fragments that compromise endothelial barrier function. Controls were immunoprecipitates of full-length netrin-1 or MMP-9 alone immunoprecipitated with netrin- $1 \mathrm{Ab}$, and they showed no effect (Figure $7 \mathrm{~B}$ ).

To validate our findings in vivo, we performed an auricular Miles assay with digested full-length netrin-1 (MMP-9-digested), VEGF, or vehicle (IP elution buffer) (Figure 7C). Our results confirmed that MMP-9-mediated digestion of full-length netrin-1 generated bioactive fragments with 3-fold greater potency in inducing vascular permeability than did the vehicle control, albeit less effectively than did VEGF (1.000 [vehicle], $3.166 \pm 0.4376$ [VI-V fragments], $P<0.05 ; 7.349 \pm 0.644$ [VEGF], $P<0.001$ ) (Figure 7D).

Given the potent effects of the VI-V fragment on vascular permeability, we sought to prevent its generation by inhibiting collagenases. At 6 and 7 weeks after induction of diabetes, we performed intravitreal injections of a soluble synthetic pan-inhibitor of collagenases (Z-Pro-D-Leu-D-Ala-NHOH; final vitreous concentration of $1 \mu \mathrm{M}$ ) (Figure 7E). This approach led to a significant reduction in diabetes-induced permeability of approximately $31 \%$ at 8 weeks of diabetes (collagenase pan-inhibitor, $31.11 \% ; 1.000 \pm$ 0.0903 [STZ], $0.6869 \pm 0.0881$ [anti-collagenase and STZ], $P<$ $0.05, n=15$ mice) (Figure $7 \mathrm{~F}$ ).
To assess the impact of MMP-9 inhibition on retinal permeability in diabetic mice, we used an anthranilic acid-based inhibitor of MMP-9 (Type I MMP-9 inhibitor; Calbiochem) (41). Intravitreal injections of the inhibitor at 6 and 7 weeks of diabetes (final vitreous concentration of $5 \mathrm{nmol} / \mathrm{l}$ ) led to a significant reduction of diabetes-induced permeability by approximately 39\% (MMP-9 inhibitor 39.11\%; $1.000 \pm 0.1050$ [STZ], $0.6089 \pm 0.1002$ [treated STZ], $P<$ $0.05, n=12$ mice) (Figure $7 G$ ). Importantly, Western blot analysis revealed that inhibition of MMP-9 reduced the levels of VI-V fragments in diabetic retinae (Figure $7 \mathrm{H}$ ) by approximately $40 \%(1.000$ \pm 0.098 [STZ], $0.585 \pm 0.1083$ [treated STZ], $P<0.05$ ) (Figure 7I). Together, these results suggest that inhibition of collagenases that generate the VI-V fragment reduces diabetes-induced retinal edema.

The VI-V fragment activates FAK and SRC kinases through UNC5B. In light of the propensity of the VI-V fragment to compromise barrier function in vivo and in culture models, we sought to determine whether the fragment triggered the signaling pathways known to influence vascular permeability. We first explored whether the VI-V fragment regulated retinal VEGF. We found that intravitreal injection of the VI-V fragment did not influence VEGF expression, suggesting an independent mode of action (1.000 \pm 0.1740 [vehicle], $0.8510 \pm 0.076$ [VI-V fragment treatment], NS) (Figure 8A).

We therefore investigated focal adhesion kinase (FAK) and SRC activation due to their implication in VE-cadherin phosphorylation (Figure 8D). Western blot analysis of HRMEC lysates after stimulation with netrin-1 $(3 \mathrm{nM})$ or the VI-V fragment $(3 \mathrm{nM})$ revealed different kinetics of VE-cadherin (Figure 8B), SRC (Figure 8B), and FAK phosphorylation (Figure 8B). Full-length netrin-1 induced rapid, maximal phosphorylation at 2.5 minutes ([p-VEcadherin/ $\beta$-actin] $0 \mathrm{~min}: 1.000 \pm 0.848 ; 2.5 \mathrm{~min}: 2.552 \pm 0.435$, $P<0.05$. [p-SRC/ $\beta$-actin] 0 min: $1.000 \pm 0.104 ; 2.5$ min: $1.506 \pm$ $0.106, P<0.05$. [p-FAK $/ \beta$-actin] 0 min: $1.000 \pm 0.089 ; 2.5$ min: $2.888 \pm 0.136, P<0.001 ; 5 \mathrm{~min}: 2.373 \pm 0.289, P<0.05)$, whereas the VI-V fragment induced delayed yet sustained phosphorylation of these pathways, peaking at around 15 to 20 minutes ([p-VEcadherin/ $\beta$-actin] $0 \mathrm{~min}: 1.000 \pm 0.081,10 \mathrm{~min}: 2.373 \pm 0.235$, $P<0.01 ; 20 \mathrm{~min}: 2.491 \pm 0.129, P<0.001$, [p-SRC/ $\beta$-actin] 0 min: $1.000 \pm 0.1177 ; 10 \mathrm{~min}: 2.581 \pm 0.178, P<0.01$, [p-FAK $/ \beta$-actin] 0 min: $1.000 \pm 0.088 ; 5 \mathrm{~min}: 4.522 \pm 0.758, P<0.01 ; 10 \mathrm{~min}: 4.728 \pm$ $0.621, P<0.01 ; 20$ min: 6.118 $\pm 0.911, P<0.01$ ) (Figure 8C).

The VI-V fragment of netrin-1 binds and signals through UNC5B. We next investigated which receptor can transduce the VI-V signal. Expression of the netrin receptors $U N C 5 B, D C C$, neogenin, and $A D O R A 2 B$ was detected in brain and retina, while DCC was absent from HRMECs (Figure 9A). We therefore individually silenced $U N C 5 B$, neogenin, or ADORA2B with siRNAs in HRMECs and compared the phosphorylation profiles of FAK, SRC, and VE-cadherin after stimulation by netrin-1 or the VI-V peptide. Transfection efficiency was evaluated with a fluorescence-labeled nontargeting siRNA control (siGLO) and assessed by FACS analysis (transfection efficiency of 80\%; siNEG, $3.053 \pm 0.5318$, siGLO, $78.93 \pm 2.898$, $P<0.0001)$. (Supplemental Figure 2, A and B). In all conditions, more than $80 \%$ of HRMECs became fluorescent, attesting to the efficiency of the approach (Supplemental Figure 2B). Silencing of each target was then verified by qPCR (Supplemental Figure 2C).

Western Blot analysis demonstrated that the silencing of UNC5B in HRMEC monolayers blocked VI-V-induced phosphor- 
A

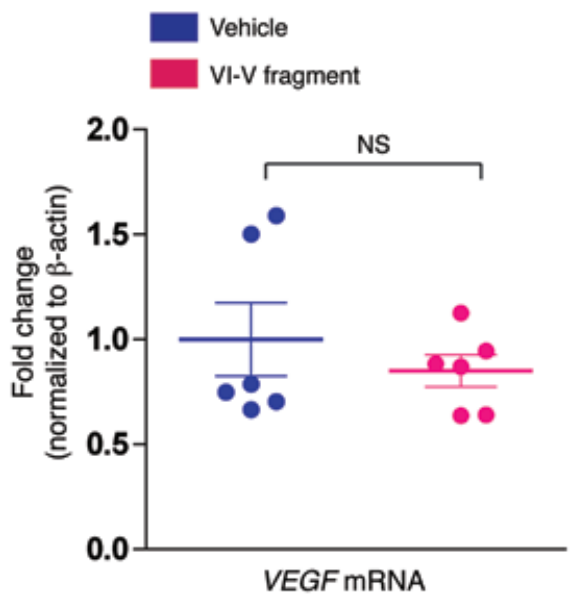

B

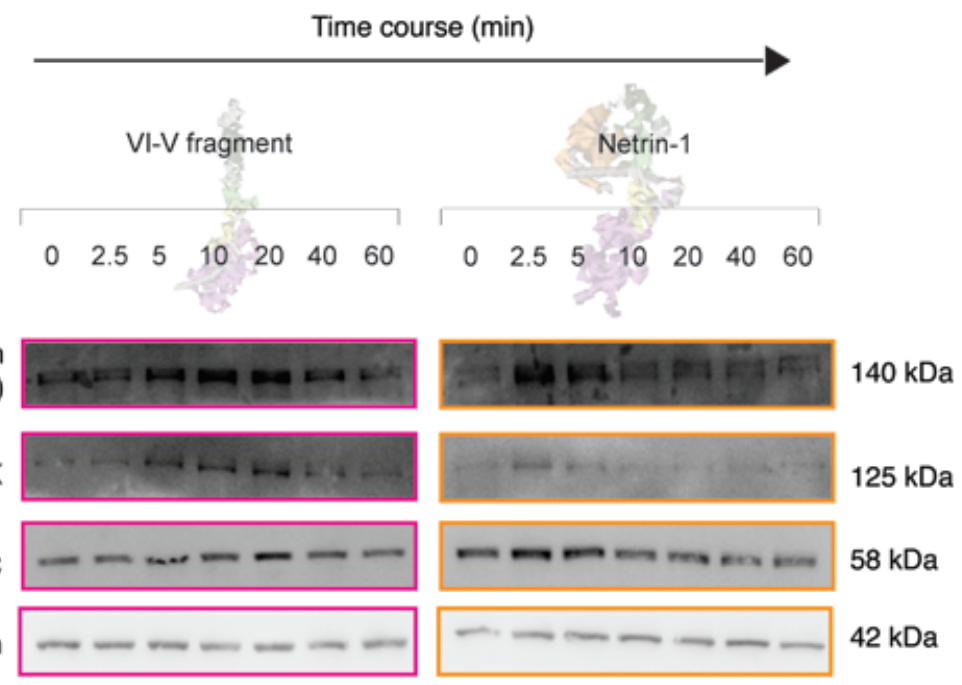

C Fold change normalized to vehicle
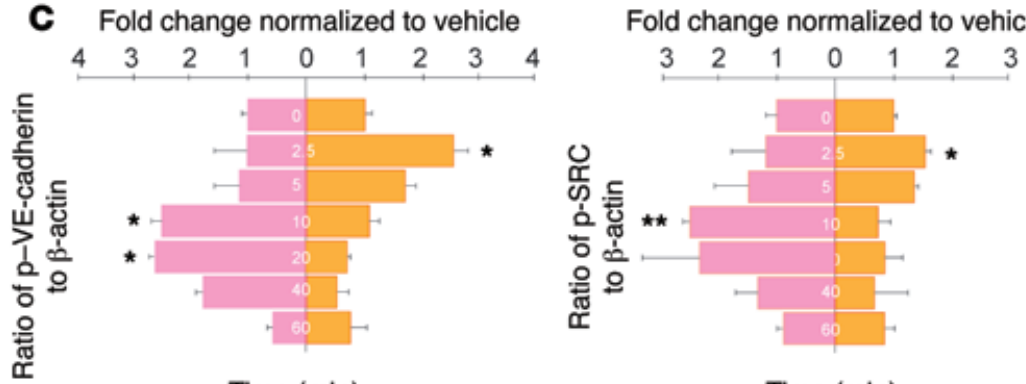

D

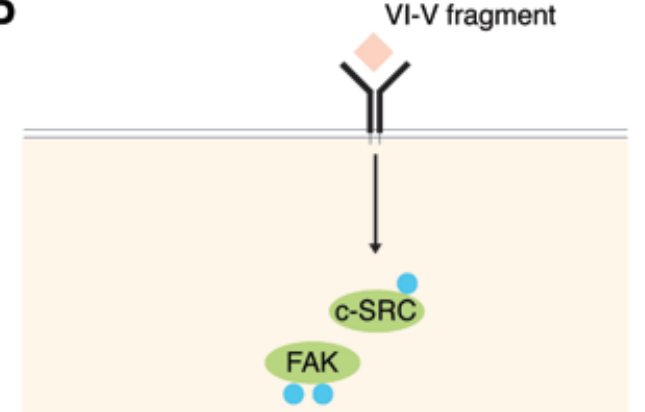

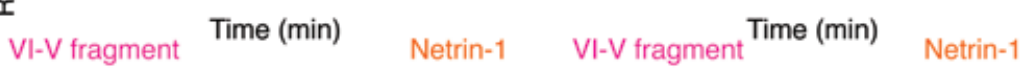

Fold change normalized to vehicle
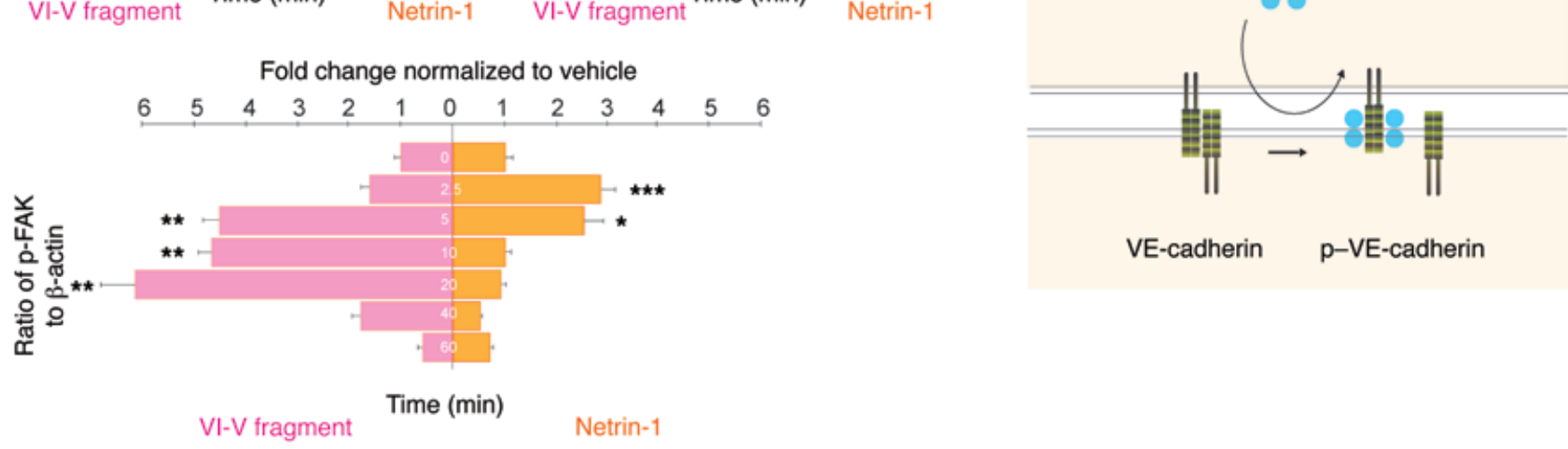

Figure 8. The VI-V fragment of netrin-1 activates FAK and SRC kinases. (A) VEGF mRNA levels after VI-V fragment intravitreal injection, NS ( $n=6$ ). (B) Representative Western blot of HRMEC treatment with VI-V fragment leading to phosphorylation of SRC, FAK, and, ultimately, VE-cadherin, with a maximum response amplitude at approximately 10 minutes, while treatment with netrin-1 showed a maximum response amplitude at 2.5 minutes $(n=3)$. (C) Quantification of $p$-SRC, p-FAK, p-VE-cadherin, and $\beta$-actin signal density in double-scale graphics, which represent $p$-SRC/ $\beta$-actin, $p$-FAK/ $\beta$-actin, and $p-V E$-cadherin/ $\beta$-actin at $0,2.5,5,10,20,40$, and 60 minutes $(n=3)$. (D) Working hypothesis. Data are expressed as the mean \pm SEM. ${ }^{*} P<0.05$, ${ }^{*} P<0.01$, and ${ }^{* *} P<0.001$, by 2 -tailed Student's $t$ test $(\mathbf{A})$ or 1 -way ANOVA with Tukey's post-hoc test (C).

ylation of VE-cadherin, whereas silencing of neogenin or ADOR$A 2 B$ had no effect (untreated: $1.000 \pm 0.052$; netrin-1-treated: siNEG, $3.448 \pm 0.363, P<0.001$, siUNC5B, $1.710 \pm 0.111$, NS; siNEO1, $3.394 \pm 0.251, P<0.001$; siADORA2B, $3.223 \pm 0.216$, $P<0.01$; VI-V fragment-treated: nontargeting control siRNA [siNEG], 3.502 $\pm 0.324, P<0.001$; siUNC5B, $2.377 \pm 0.157$, NS; SiNEO1, $4.112 \pm 0.589, P<0.001$; siADORA2B, $4.355 \pm 0.641, P$ $<0.001$ ). (Figure 9, B and C). These results were corroborated by ECIS experiments, which showed that silencing the UNC5B receptor in endothelial cell monolayers rescued VI-V-induced permeability (relative resistance normalized to siNEG plus vehicle) (Figure 9D).
IHC and 3D retinal reconstruction (Figure 9E) confirmed that UNC5B was predominantly expressed in mature vasculature. We then used LCM to measure the expression of UNC5B in the different retinal layers and in the vasculature. Our data show that UNC5B expression was upregulated ( 4-fold increase) in STZinduced diabetic vasculature compared with expression in control mice that were only injected with citrate (citrate, $1.000 \pm 0.342$, STZ, 3.954 $\pm 0.6241, P<0.05$ ) (Figure 9F).

Given the discrepancy in the activation kinetics of netrin- 1 and its VI-V fragment to activate permeability pathways (Figure 8), we next investigated the binding affinity toward UNC5B of full-length netrin-1 and the VI-V fragment via surface plasmon resonance 
A

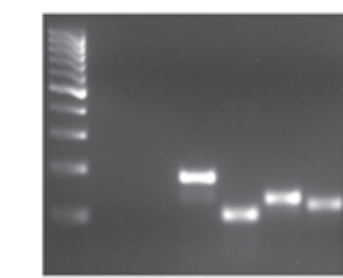

$18 \mathrm{~S}$
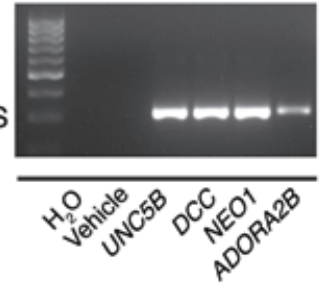

D

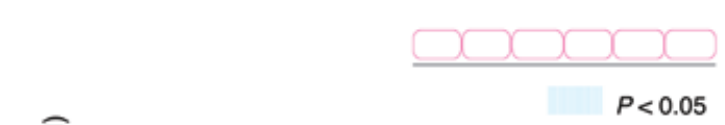

Retina
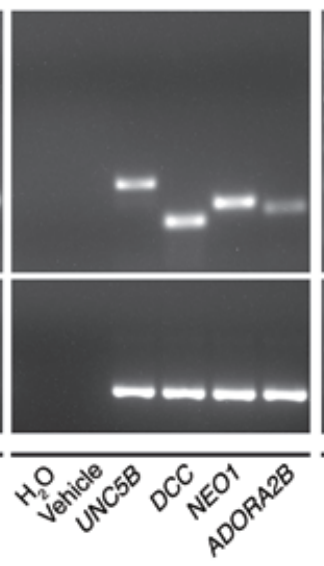

HUVECS
HRMECs
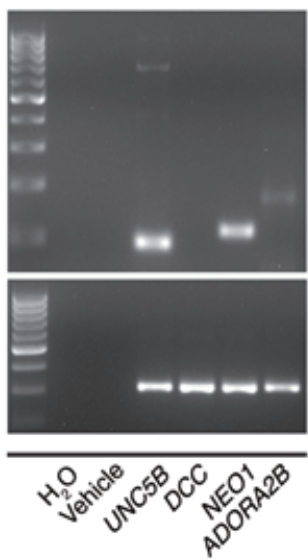

$000 \mathrm{~Hz}$

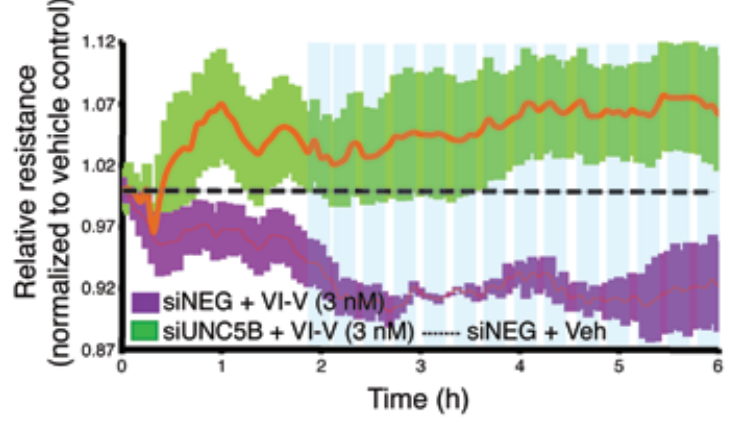

E
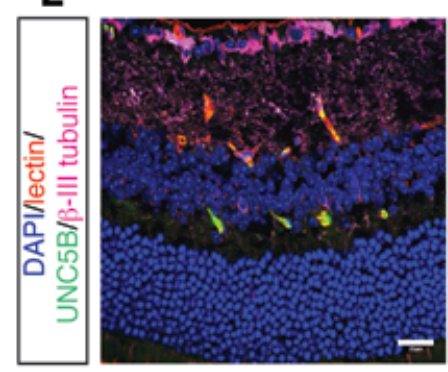

B

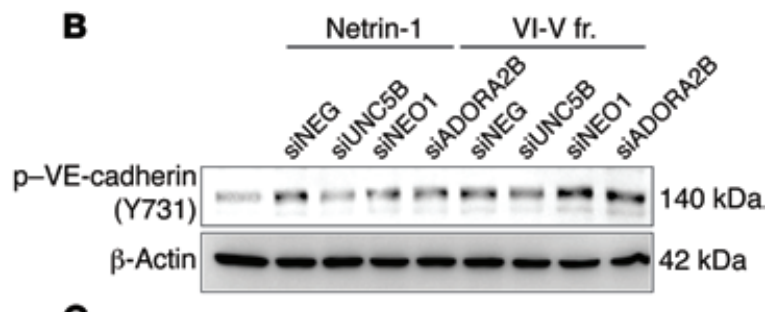

C

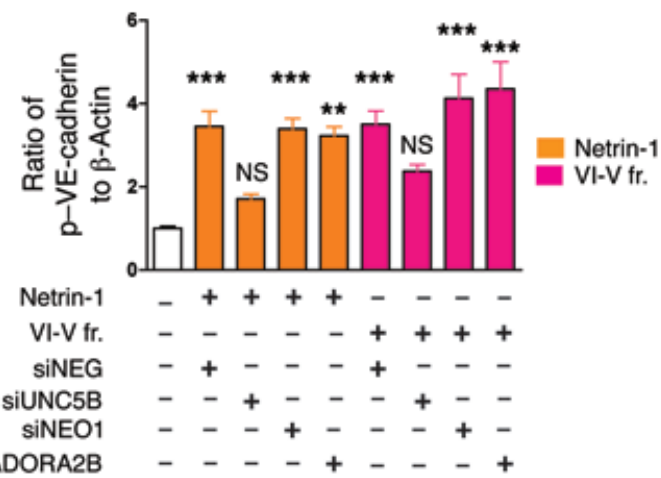

UNC5B-FC immobilized
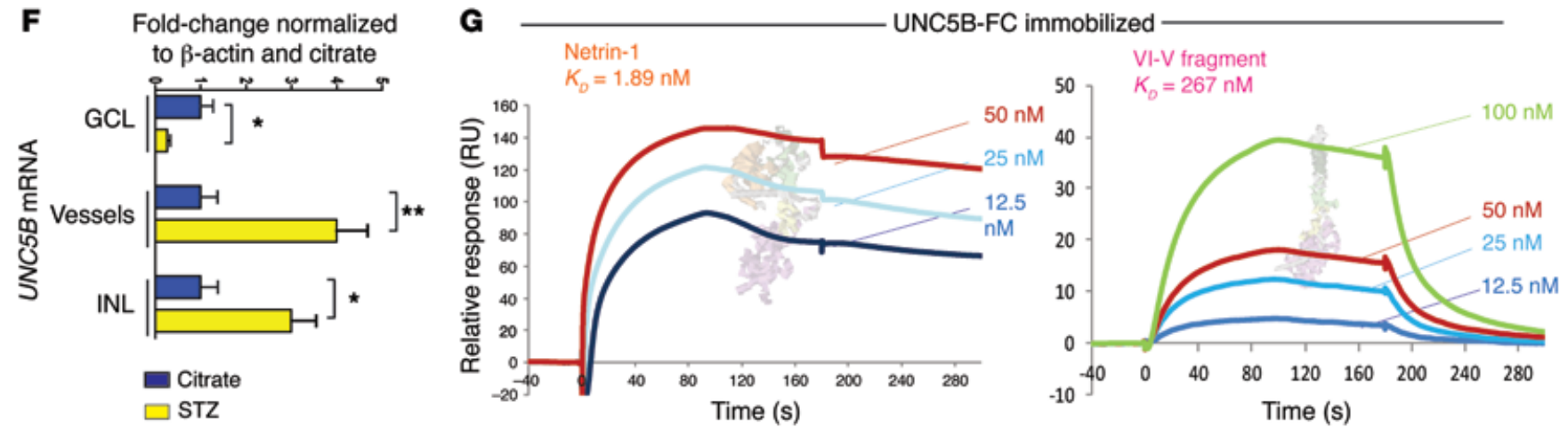

Figure 9. The VI-V fragment of netrin-1 binds and signals through UNC5B. (A) UNC5B, DCC, NEO1, and ADORA2B expression in endothelial cells ( $n=3$ ). (B) Representative Western blot showing loss of p-VE-cadherin, with silencing of the UNC5B receptor $(n=3)$. (C) p-VE-cadherin blot quantification ( $n=3$ ). (D) Paracellular resistance measured in real time by ECIS demonstrated that the VI-V fragment ( $3 \mathrm{nM}$ ) did not compromise endothelial barrier function in endothelial cells transfected with siUNC5B compared with those transfected with siNEG (1.8-6 hours, $n=4)$. (E) Representative confocal images of isolectin B4- (vessels), $\beta$-III tubulin- (retinal ganglion cells [RGCs]), DAPI- (nuclei), and UNC5B-stained retinal cross sections of adult mice revealed that retinal UNC5B was essentially vascular $(n=3)$. Scale bar: $20 \mu \mathrm{m}$. (F) LCM of the GCL, vessels, and INL, followed by qPCR of UNC5B $(n=3)$. (G) Binding strength of full-length netrin-1 versus that of the VI-V fragment to UNC5B via SPR $(n=4)$. FC, fragment crystallizable region. Data are expressed as the mean \pm SEM. ${ }^{*} P<0.05,{ }^{* *} P<0.01$, and ${ }^{* *} P<0.001$, by 1-way ANOVA with Tukey's post-hoc test (C) or 2-tailed Student's $t$ test (D and $\left.\mathbf{F}\right)$.

(SPR). We found that the affinity of the VI-V fragment was approximately 100-fold lower than that of netrin-1 (netrin- $1 K_{D}=1.9 \mathrm{nM}$, versus VI-V fragment $K_{D}=267 \mathrm{nM}$ ) (Figure 9G). Kinetics analysis of the full-length netrin-1-UNC5B association also revealed a rapid association and slow dissociation, which is consistent with the VE-cadherin phosphorylation patterns observed at early time points (2.5 and $5 \mathrm{~min}$ ) (Figure 8B). Slow dissociation may suggest that full-length netrin-1 remains bound to UNC5B and reinforces barrier function, as has been suggested for UNC5B-roundabout guidance receptor 4 (UNC5B-ROBO4) interactions (42).

SPR experiments also revealed that netrin- 1 was tightly associated with the negatively charged carboxymethylated dextran surface of the sensor chip in contrast to the VI-V fragment, which showed low, nonspecific binding to the dextran matrix. By using sensor chips with different charge densities and/or chain lengths (CM4, CM3, CM5), we observed that the nonspecific interactions 

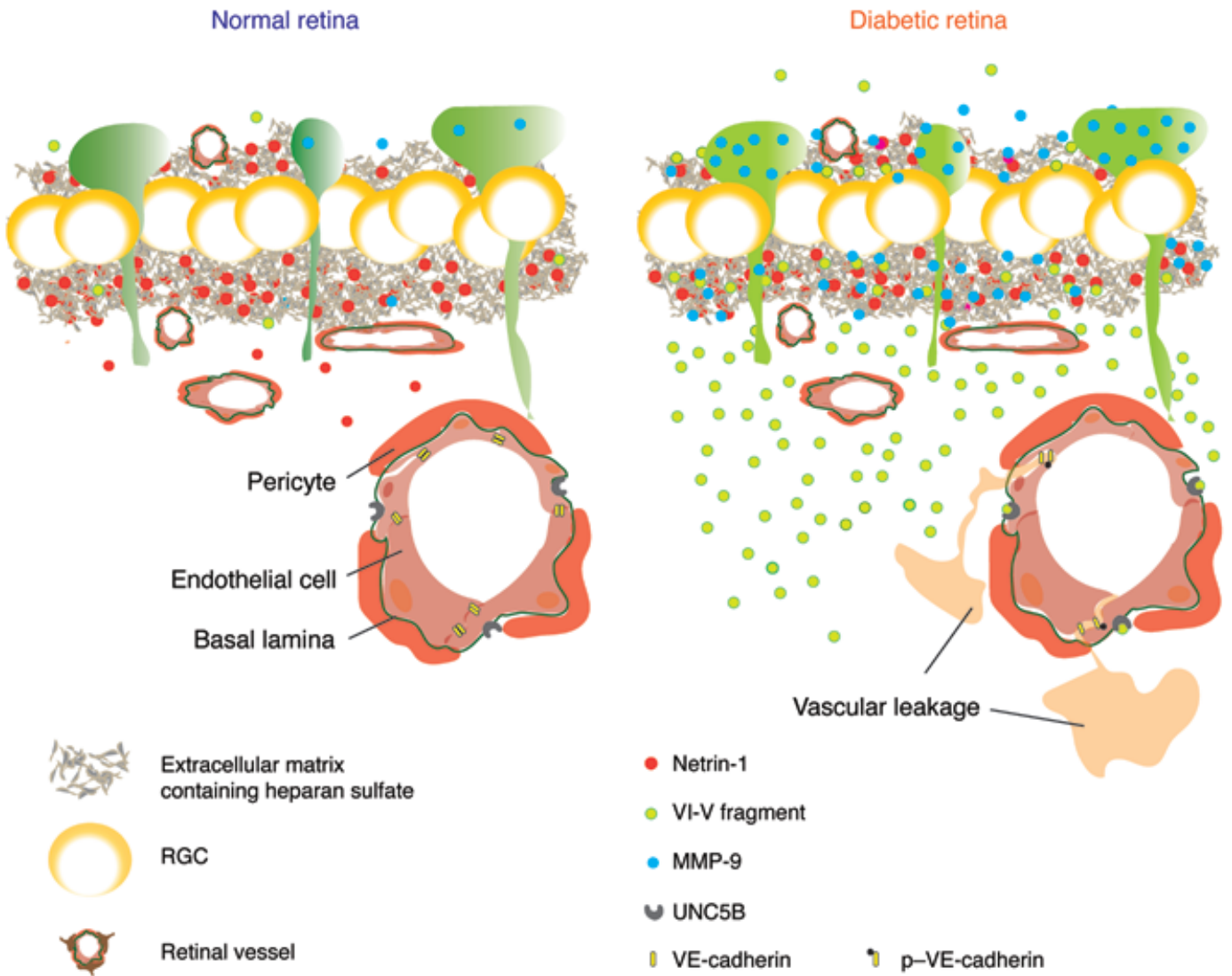

Müller cell

Figure 10. Schematic mechanism of full-length netrin-1 processing by MMP-9-inducing vascular permeability.

of netrin-1 were driven by electrostatic interactions between the positively charged NTR domain and the carboxylated dextran. These observations could be interpreted as an echo of the real bioavailability of netrin- 1 in a healthy retina or in a diabetic retina, where a pool of netrin- 1 could reside in the negatively charged heparin sulphate proteoglycans of the GCL (43). Cleavage of netrin-1 via MMP-9 or other collagenases in DR would release the VI-V fragment to carry out its physiological functions (Figure 10).

\section{Discussion}

A mechanistic understanding of the pathogenesis of DR and DME has evolved in recent years beyond microvessels and now encompasses early disturbances in the neurovascular unit as a whole (2). This view of the disease is particularly plausible, given the preponderance of neural (neuronal and glial) cells versus endothelial and mural cells in the retina and the substantial metabolic requirements of neuronal transmission (44). In this study, we provide evidence that DR is associated with the processing of the guidance cue netrin- 1 , a protein with the multivalent ability to influence vascular, neuronal, and immune systems (37). Contrary to several other guidance cues, expression of netrin-1 is not limited to embryogenesis, and its levels remain elevated in mature retina (Figure 2), suggesting a role in tissue homeostasis. We demonstrate that during diabetes, retinal netrin- 1 is processed by
MMP-9 and other collagenases into the more soluble VI-V fragment. Although future work will identify the exact sequence of the netrin fragment detected in retinopathy, the current study supports the conclusion that the $55-\mathrm{kDa}$ fragment observed in vitreous samples of DME patients and in the diabetic mouse retina is similar to the VI-V fragment.

While full-length netrin-1 has vasoprotective properties (21) and maintains blood-brain barrier function (45), the newly generated fragments contribute to the breakdown of the BRB and thus exacerbate retinal and macular edema. A role for the VI-V fragment of netrin-1 in pathology was previously suggested, given its presence in multiple sclerosis (MS) plaques and capacity to inhibit oligodendrocyte migration (46). While netrin-1 is required for the maintenance of axo-oligodendroglial paranodal junctions (47), ectopic expression of netrin-1 in vivo inhibited remyelination in an experimental model of MS lesions (48).

Retinal netrin- 1 is produced primarily in the GCL adjacent the retinal vascular plexus affected in DR. Paradoxically, at similar time points, retinal ganglion neurons in diabetes also secrete the guidance cue semaphorin $3 \mathrm{~A}$, which is a potent inducer of vascular permeability (17). However, degradation of netrin-1 by collagenases and MMPs generates fragments, such as the VI-V peptide, that exacerbate retinal edema. Together, these data further suggest that cells of the ganglion layer undergo diabetes-induced 
stress and produce vasomodulatory proteins that are involved in the mediation of vascular phenotypes in DR.

The present study also provides evidence for a diabetesinduced upregulation of the netrin-1 receptor UNC5B in retinal microvessels. These findings are consistent with those of several studies describing elevated levels of UNC5B in conditions associated with chronic inflammation such atherosclerosis, with induction of UNC5B in plaques (49), obesity, with upregulation of UNC5B in macrophages (31), and oxygen-induced retinopathy, with induction of UNC5B in retinal vessels (50). Consistent with our findings, a role for UNC5B in vascular integrity had previously been demonstrated, in which interaction between UNC5B and the extracellular domain of the ROBO4 receptor prevents VEGFdriven permeability (42). Hence, induction of UNC5B on retinal microvessels may enhance vascular integrity and function as a protective mechanism of adaptation to diabetes. Activation of UNC5B via the VI-V fragment of netrin-1 may disrupt the vasostabilizing interactions of UNC5B and thereby promote vasogenic edema.

Our results point to apparently divergent effects of full-length netrin- 1 and the VI-V fragment on the vasculature. The lower binding affinity of the VI-V fragment versus that of full-length netrin-1 with UNC5B may explain the differential FAK, SRC, and VE-cadherin phosphorylation kinetics observed. Yet, full-length netrin-1 dissociates less readily, suggesting a possible sequestration by the receptor. MMP-9-mediated cleavage of netrin-1 generates fragments with opposite actions, much like what has been described for cytokines (51). MMP-9-mediated proteolysis of netrin-1 may also increase the bioavailability of netrin-1, akin to VEGF distribution in tissue (52). The resulting fragments would be liberated to diffuse within the retina and increase vasopermeability.

The landscape for the treatment of DME evolved with the introduction of anti-VEGFs such as bevacizumab (Avastin), ranibizumab (Lucentis), and aflibercept (Eylea). Paradoxically, their ability to effectively block VEGF function may compromise retinal function in the long term, given the fundamental role of VEGF as a neurotrophic factor $(12,13)$. Because VEGF is not upregulated in the early phases of disease (17), blocking the biological activity of the VI-V fragment may be of therapeutic interest. Given that the VI-V fragment and full-length netrin- 1 can both signal through UNC5B (other receptors cannot be excluded), that the UNC5B receptor is required for vascular stability (42), and considering the vasoprotective role of netrin-1 $(21,45)$, a successful approach may require interfering with the processing of full-length netrin-1. The positively charged NTR module has been observed to interact with extracellular matrix heparin sulfates (53) and has also been reported to control MMP activity (54). Given the induction of MMPs such as MMP-9 in diabetic retinae and their contribution to generation of the VI-V fragment, neutralizing MMPs (55) may also provide prospective pharmacological avenues once specific inhibitors are generated. To date, broad-spectrum MMP inhibitors have failed in oncology trials, largely because of the crucial roles of MMPs in several ubiquitous cellular and extracellular processes such as cell-surface receptor cleavage and release as well as cytokine and chemokine activation and inactivation (56). Further research is still needed to develop potent selective MMP inhibitors that will allow for successful clinical applications (57).

Collectively, our results demonstrate that processing of netrin- 1 in the diabetic retina leads to vasoactive fragments with the capacity to exacerbate vasogenic edema. While future studies will determine the therapeutic merits of interfering with netrin-1 fragmentation, our study provides further evidence for the role of guidance cues and neurovascular interplay in DR.

\section{Methods}

\section{Animals}

C57BL/6 WT mice were purchased from the Jackson Laboratory and CD-1 mice from Charles River Laboratories.

\section{Vitrectomy}

All patients previously diagnosed with DME were followed up and operated on by a single vitreoretinal surgeon (FAR). The control patients were undergoing surgical treatment for nonvascular pathology (epiretinal membrane [ERM] or macular hole) by the same surgeon. Topical instillation of $5 \%$ povidone-iodine solution was applied to the ocular surface and used to clean the periocular skin prior to the surgical procedure. For sampling of DME vitreous, patients were then draped in a standard sterile manner with placement of a lid speculum. A 27-gauge self-retaining line (Insight Instruments) for balanced salt solution (BSS) infusion was first placed, followed by a 29-gauge chandelier placement connected to a mercury vapor light source (Synergetics). The surgical view during the procedure was provided through a surgical operative microscope and a Volk contact lens (Volk direct image $\times 1.5$ magnifying disposable vitrectomy lens). The vitrectomy was performed in all patients using a 25-gauge sutureless retractor system (Insight Instruments). The model used in the study is a portable, battery-powered system with a maximum cut rate of $600 \mathrm{cpm}$ and features a single-use retractable sheathed guillotine cutter (25-gauge) with an in-built needle (23-gauge). The needle was introduced bevel down through displaced conjunctiva in an oblique single-plane tunnel into the vitreous cavity $3-4 \mathrm{~mm}$ from the limbus. With the exception of the portable vitrector motor handpiece, which was placed within a sterile plastic cover when in use, all other instruments used were sterile and disposable. For patients with ERM and macular hole (controls), a standard 25-gauge 3-port pars plana vitrectomy was performed in the operating room.

\section{$3 D$ rendering of the netrin-1 structure}

The Robetta 3D structure server (58) was used to generate full-chain protein structures of full-length netrin-1 and of the VI-V fragment. Prediction was performed using the Ginzu protocol, consisting of ab initio fragment assembly. Ginzu identifies homologs with an experimentally determined structure (pdb blast-p), generates a multiple alignment of the highest-scoring pairs of the blast-p, and ultimately selects cut-points between domains (PSI Blast MSA).

\section{Diabetic mouse mode}

Multiple low doses of STZ (Sigma-Aldrich) were administered i.p. on 5 consecutive days to 6-week-old C57BL/6J mice at a concentration of $55 \mathrm{mg} / \mathrm{kg}$ to induce a type 1 diabetic model. Control mice were injected i.p. with sodium citrate buffer.

\section{Cell lines}

HRMECs were purchased from Cell Systems and HUVECs from Lonza and were used from passages 6 to 11. HRMECs were cultured 
in EGM-2 microvascular medium from Lonza, whereas HUVECs were cultured in EGM-2 macrovascular medium from Lonza. For stimulation experiments and for real-time ECIS, cells were previously starved for 4 hours with EBM-2 medium from Lonza plus 0.5\% FBS. Recombinant VEGF ${ }_{165}$ was purchased from Peprotech.

\section{Recombinant truncated netrin-1 VI and V}

Truncated netrin-1 containing domains VI and V with a C-terminal Myc tag was generated by expression in Epstein-Barr virus nuclear antigen/human embryonic kidney 293 (EBNA 293) cells with the use of the pCEP4 vector (Invitrogen) and purified by a heparin sepharose high-performance column (HR 5/10; Amersham Biosciences).

\section{Western blot analysis}

For assessment of retinal protein levels, we enucleated eyes at 4,6 , and 8 weeks of diabetes (versus citrate controls) and rapidly dissected and homogenized retinae. Protein concentration was assessed by bicinchoninic acid (BCA) assay (Sigma-Aldrich), and $30 \mu \mathrm{g}$ protein was analyzed for each condition by standard SDS-PAGE technique. For supernatants, we loaded equal volumes of samples on gels and determined the amount of protein present by Ponceau red (Sigma-Aldrich) staining of membranes.

Netrin-1 rabbit polyclonal Abs against netrin-1 VI-V antigen were generated as described previously (59). MMP-9 Ab was purchased from Abcam (catalog ab119906). Anti-VE-cadherin Ab (p-Y731) was purchased from Abcam (catalog ab2776). p-FAK (Tyr 576/577) and p-SRC (Tyr 16) Abs were purchased from Cell Signaling Technology. $\beta$-Actin was purchased from Medimabs (catalog MM-0164P).

\section{Netrin-1 processing in vitro}

Netrin-1 fragments were generated in vitro as follows: $3 \mathrm{nM}$ synthetic netrin- 1 was digested in $100 \mu \mathrm{l}$ of active MMP-9 $(1 \mu \mathrm{g})$ from Abcam (catalog ab168863) for 30 minutes at $37^{\circ} \mathrm{C}$. The reaction was then stopped with proteases inhibitors and the mix stored at $-80^{\circ} \mathrm{C}$ for subsequent Western blot analysis and IP. For mass spectrometric analysis, we used a larger quantity of netrin-1 $(10 \mu \mathrm{g})$.

\section{Netrin-1 IP}

Netrin-1 mAb (ALX-804-838-C100, clone NORA-1) used for IP was purchased from Enzo (Enzo Life Sciences). To avoid contamination of the $\mathrm{Ab}$ for permeability assays, we used a Pierce Crosslink IP Kit (Thermo Fisher Scientific).

\section{qPCR}

Extraction of mRNA was performed by standard TRIzol extraction. DNase digestion to prevent amplification of genomic DNA was then performed and was followed by a retrotranscription step to generate cDNA using a Bio-Rad iScript cDNA Synthesis Kit. Gene expression was then quantified using SYBR Green in an ABI Biosystems RealTime PCR system. Actb was used as a reference gene.

\section{ECIS assay}

Real-time analysis of trans- and interendothelial impedance was performed by plating $10^{5}$ HRMECs and HUVECs onto 8W10E+ 8-well arrays (Applied Biophysics) (40 electrodes per well). Cells were allowed to grow until resistance stabilized at approximately 2,000 $\Omega$. Cells were then starved for 4 to 6 hours in basal medium (EBM2) and stimulated for 10 hours. The results were then normalized to the vehicle control and expressed as relative resistance.

\section{LCM}

Eyes were enucleated from C57BL/6J mice at 4 and 8 weeks of diabetes (versus citrate controls) and flash frozen in OCT. We then cut $12-\mu \mathrm{m} \mathrm{sec}-$ tions using a Leica cryostat at $-20^{\circ} \mathrm{C}$ and air dried the sections for $10 \mathrm{~min}$ utes. For LCM, retinal layers were dissected using a Zeiss Observer microscope equipped with a Palm MicroBeam device. mRNA was isolated from these sections and qPCRs were performed as described above.

\section{Mass spectrometry}

One hundred microliters of full-length netrin-1 and digested netrin-1 was dried in a Speed-Vac (Thermosavant), resolubilized in $0.2 \%$ formic acid (FA), and analyzed on an Agilent 1100 LC coupled to an Agilent 6510 Q-TOF instrument. Samples were loaded on a C5 Phenomenex precolumn $(360 \times 500 \mu \mathrm{m})$ and separated on an anatycal column $(150 \mu \mathrm{m} \times 15 \mathrm{~cm})$, with a 56 -minute gradient from $0 \%$ to $30 \%$ acetonitrile $(0.2 \% \mathrm{FA})$ and a $600 \mathrm{nl} /$ minute flow rate.

\section{SPR}

SPR analyses were performed using a Biacore T200 instrument (GE Healthcare). UNC5B recombinant protein was immobilized on carboxymethylated dextran CM5 (for VI-V) and CM4 (for netrin) sensor chips (GE Healthcare) using an amine-coupling strategy. Briefly, the sensor chip surface was activated with a 1:1 mixture of $N$-hydroxysuccinimide and 3-( $N, N$-dimethylamino)-propyl- $N$-ethylcarbondiimide. UNC5B solution (solubilized in acetate buffer, $\mathrm{pH} 4.5$ ) was injected at a flow rate of $20 \mu \mathrm{l} /$ minute in PBS running buffer ( $\mathrm{pH} 7.2 ; 0.025 \%[\mathrm{v} / \mathrm{v}]$ Tween-20) to reach a level of immobilization of 400 and 800 relative units (RU) on CM5 and CM4 sensor chips, respectively. Surfaces (protein and reference) were blocked by the injection of an ethanolamine solution. Binding kinetics of netrin and VI-V over the UNC5B sensor chip was evaluated in PBS buffer ( $\mathrm{pH} 7.2 ; 0.025 \%$ [v/v] Tween-20), with concentrations ranging from 12.5 to $100 \mathrm{nM} \mathrm{VI-V}$ and netrin, respectively. All tests were performed at $25^{\circ} \mathrm{C}$ using a flow rate of 40 $\mu \mathrm{l} /$ minute. Sensor chip surfaces were regenerated by twice injecting $20 \mu \mathrm{l}$ of a $50 \mathrm{mM} \mathrm{NaOH}$ solution at a flow rate of $40 \mu \mathrm{l} /$ minute. Binding sensograms were obtained by subtracting the reference flow cell. Data analysis was performed using BIA Evaluation Software (GE Healthcare) and fit to a one-site Langmuir binding model.

\section{Collagenase and MMP-9 inhibitors}

Collagenase inhibitor (EMD Millipore Calbiochem; catalog CAS 234140) was injected intravitreally into diabetic C57BL/6J mice at 6 and 7 weeks of diabetes. Type I MMP-9 inhibitor (EMD Millipore Calbiochem; catalog CAS 11777 49-58-4) was injected in the same way into diabetic mice at 6 and 7 weeks of diabetes. For both experiments, $1 \mu \mathrm{l}(5 \mu \mathrm{M}$ for the collagenase inhibitor, $25 \mathrm{nM}$ for the MMP-9 inhibitor) was injected into the vitreous.

\section{Permeability assays}

We performed 3 different tests of permeability, all based on fluorescent dye extravasation.

Retinal EB permeation assay. EB ( $45 \mathrm{mg} / \mathrm{kg}$; Sigma-Aldrich) was injected i.v. into C57BL/6J mice and allowed to circulate for 2 hours. Eyes were then enucleated, fixed for 1 hour in $4 \%$ paraformaldehyde 
(PFA), and the retina dissected. EB was extracted overnight in formamide at $65^{\circ} \mathrm{C}$ and quantified by spectrophotometry $(620 \mathrm{~nm}$ maximum, $740 \mathrm{~nm}$ minimum absorbance) (17).

Auricular Miles assay. EB (45 mg/kg) was injected i.v. into CD-1 mice. After 10 minutes, the mice were injected with PBS (vehicle) into the control ear, and PBS VI-V (3-7 nM), PBS VEGF (2 nM), or PBS purified fragments $(\sim 3 \mathrm{nM})$ into the contralateral ear. EB extravasation was photographed 3 hours after EB injection and evaluated by Image J software (NIH) (60).

Modified Miles assay. EB ( $45 \mathrm{mg} / \mathrm{kg})$ was injected i.v. into C57BL/6 mice. After 10 minutes, the mice were injected s.c. with PBS (vehicle), PBS VI-V fragment (3 nM), or PBS VEGF ( $2 \mathrm{nM})$. EB extravasation was evaluated 3 hours after EB injection.

Retinal angiography was performed as previously described (61). Briefly, C57BL/6J mice were anesthetized, and the descending aorta was clamped and then perfused via the left ventricle with $1 \mathrm{ml}$ warm PBS containing $50 \mathrm{mg}$ high-molecular-weight $\left(2 \times 10^{6} \mathrm{Da}\right)$ FITC (Sigma-Aldrich). After 2 minutes of circulation, the eyes were enucleated and placed in 4\% PFA for 2 hours. Retinae were then dissected, stained overnight with lectin- $\mathrm{MgCl}_{2}$, and flat-mounted in fluorescent mounting medium. Leakage was observed and photographed under a confocal microscope at $488 \mathrm{~nm}(\times 10$ objective $)$.

\section{IHC}

Eyes were enucleated from mice and fixed in $4 \%$ PFA at room temperature for 4 hours, incubated in $30 \%$ sucrose overnight at $4^{\circ} \mathrm{C}$, and frozen in optimal OCT compound. Sections of $10-\mu \mathrm{m}$ thickness were then prepared using a cryostat (Leica), keeping the temperature at $-20^{\circ} \mathrm{C}$. IHC experiments were performed and cross sections visualized with an Olympus FluoView confocal microscope.

UNC5B Ab was purchased from Abcam (catalog ab54430). IBA1 Ab was purchased from Wako (catalog 019-19741). Rhodamine isolectin B4 was purchased from Vector Laboratories (catalog RL-1102). DAPI was purchased from Molecular Probes (Thermo Fisher Scientific).

\section{Statistics}

Data are presented as the mean \pm SEM. One-way ANOVA with Tukey's post-hoc tests were used to compare differences between groups. A 2 -tailed Student's $t$ test was used when only 2 groups were compared. The criterion for statistical significance was a $P$ value of less than 0.05.

Study approval. Approval of the human clinical protocol was provided by the ethics committee of Maisonneuve-Rosemont Hospital (HMR) (Ref. CER: 10059), and informed consent was obtained from patients with type 2 diabetes mellitus, who were recruited for local core vitreal biopsy sampling. The entire procedure was performed on an outpatient basis in the minor procedure room within the ambulatory clinic of the Department of Ophthalmology at MaisonneuveRosemont Hospital. All instruments were opened and handled in a sterile manner. The study conformed to the tenets of the declaration of Helsinki and was approved by the IRB of the Maisonneuve-Rosemont Hospital affiliated with the University of Montreal.

All animal studies were performed according to the Association for Research in Vision and Ophthalmology Statement for the Use of Animals in Ophthalmic and Vision Research and were approved by the Animal Care Committee of the University of Montreal in agreement with the guidelines established by the Canadian Council on Animal Care.

\section{Author contributions}

KM designed the research studies, conducted experiments, acquired and analyzed data, and wrote the manuscript. FB and AW wrote the manuscript. AC, MO, CM, SH, GM, AD, and PTN conducted experiments. FAR provided vitreous samples. SB analyzed data. TEK provided reagents, contributed to the study design, and wrote the manuscript. PS designed the research studies and wrote the manuscript.

\section{Acknowledgments}

$\mathrm{K}$. Miloudi is supported by an operating grant from the Canadian Diabetes Association (OG-3-11-3329-PS). P. Sapieha holds the Wolfe Professorship in Translational Vision Research, a Canada Research Chair in Retinal Cell Biology, and is a recipient of the Alcon Research Institute Young Investigator Award. T.E. Kennedy was supported by a Fond de Recherches Québec Santé (FRQS) Chercheur National award and a Killam Foundation Scholar award. This work was supported by the Canadian Diabetes Association (OG-3-11-3329-PS); the Foundation Fighting Blindness Canada (FFB); the Canadian Institutes of Health Research (324573 and 221478); the Natural Sciences and Engineering Research Council of Canada (418637); and the Multiple Sclerosis Society of Canada (2120). Additional support was obtained from the Fondation HMR, Réseau en Recherche en Santé de la Vision du Québec and the Fond en Recherche en Opthalmologie de l'Université de Montréal. We would also like to thank Aouatef Benlemouden for help with clinical samples and Nathalie Marcal for technical assistance. We acknowledge Eric Bonneil from the Institut de Recherche en Immunologie et Cancérologie (IRIC) proteomic platform for mass spectrometric analyses.

Address correspondence to: Przemyslaw (Mike) Sapieha, Maisonneuve-Rosemont Hospital Research Centre, 5415 Assomption Boulevard, Montreal, Qc, H1T 2M4, Canada. Phone: 514.252.3400, ext.7711; E-mail: mike.sapieha@umontreal.ca.
1. Kempen JH, et al. The prevalence of diabetic retinopathy among adults in the United States. Arch Ophthalmol. 2004;122(4):552-563.

2. Antonetti DA, Klein R, Gardner TW. Diabetic retinopathy. NEngl JMed. 2012;366(13):1227-1239.

3. Cunha-Vaz J, Ribeiro L, Lobo C. Phenotypes and biomarkers of diabetic retinopathy. Prog Retin Eye Res. 2014;41:90-111.

4. Ciulla TA, Amador AG, Zinman B. Diabetic retinopathy and diabetic macular edema: pathophysiology, screening, and novel therapies. Diabetes Care. 2003;26(9):2653-2664.
5. Moss SE, Klein R, Klein BE. The 14-year incidence of visual loss in a diabetic population. Ophthalmology. 1998;105(6):998-1003.

6. Simó R, Hernández C. Novel approaches for treating diabetic retinopathy based on recent pathogenic evidence. Prog Retin Eye Res. 2015;48:160-180.

7. Tang J, Kern TS. Inflammation in diabetic retinopathy. Prog Retin Eye Res. 2011;30(5):343-358.

8. Antonetti DA, Lieth E, Barber AJ, Gardner TW. Molecular mechanisms of vascular permeability in diabetic retinopathy. Semin Ophthalmol. 1999;14(4):240-248.
9. Calcutt NA, Cooper ME, Kern TS, Schmidt AM. Therapies for hyperglycaemia-induced diabetic complications: from animal models to clinical trials. Nat Rev Drug Discov. 2009;8(5):417-429.

10. Hammes HP, Feng Y, Pfister F, Brownlee M. Diabetic retinopathy: targeting vasoregression. Diabetes. 2011;60(1):9-16.

11. Silva PS, Cavallerano JD, Sun JK, Aiello LM, Aiello LP. Effect of systemic medications on onset and progression of diabetic retinopathy. Nat Rev Endocrinol. 2010;6(9):494-508.

12. Robinson GS, et al. Nonvascular role for VEGF: 
VEGFR-1, 2 activity is critical for neural retinal development. FASEB J. 2001;15(7):1215-1217.

13. Saint-Geniez M, et al. Endogenous VEGF is required for visual function: evidence for a survival role on müller cells and photoreceptors. PLoS One. 2008;3(11):e3554.

14. Grunwald JE, et al. Risk of geographic atrophy in the comparison of age-related macular degeneration treatments trials. Ophthalmology. 2014;121(1):150-161.

15. Barber AJ, et al. The Ins2Akita mouse as a model of early retinal complications in diabetes. Invest Ophthalmol Vis Sci. 2005;46(6):2210-2218.

16. Barber AJ, Lieth E, Khin SA, Antonetti DA, Buchanan AG, Gardner TW. Neural apoptosis in the retina during experimental and human diabetes. Early onset and effect of insulin. JClin Invest. 1998;102(4):783-791.

17. Cerani A, et al. Neuron-derived semaphorin $3 \mathrm{~A}$ is an early inducer of vascular permeability in diabetic retinopathy via neuropilin-1. Cell Metab . 2013;18(4):505-518.

18. Dejda A, et al. Neuropilin-1 mediates myeloid cell chemoattraction and influences retinal neuroimmune crosstalk. JClin Invest. 2014;124(11):4807-4822.

19. Kern TS, Engerman RL. Capillary lesions develop in retina rather than cerebral cortex in diabetes and experimental galactosemia. Arch Ophthalmol.1996;114(3):306-310.

20. Lu X, et al. The netrin receptor UNC5B mediates guidance events controlling morphogenesis of the vascular system. Nature. 2004;432(7014):179-186.

21. Binet F, et al. Neuronal ER stress impedes myeloid-cell-induced vascular regeneration through IRE1 $\alpha$ degradation of netrin-1. Cell Metab. 2013;17(3):353-371.

22. Fukushima Y, et al. Sema3E-PlexinD1 signaling selectively suppresses disoriented angiogenesis in ischemic retinopathy in mice. J Clin Invest. 2011;121(5):1974-1985.

23. Joyal JS, et al. Ischemic neurons prevent vascular regeneration of neural tissue by secreting semaphorin 3A. Blood. 2011;117(22):6024-6035.

24. Vieira JM, Schwarz Q, Ruhrberg C. Role of the neuropilin ligands VEGF164 and SEMA3A in neuronal and vascular patterning in the mouse. Novartis Found Symp. 2007;283:230-235.

25. Adams RH, et al. Roles of ephrinB ligands and EphB receptors in cardiovascular development: demarcation of arterial/venous domains, vascular morphogenesis, and sprouting angiogenesis. Genes Dev. 1999;13(3):295-306.

26. Wang HU, Chen ZF, Anderson DJ. Molecular distinction and angiogenic interaction between embryonic arteries and veins revealed by ephrin-B2 and its receptor Eph-B4. Cell. 1998;93(5):741-753.

27. Park KW, et al. Robo4 is a vascular-specific receptor that inhibits endothelial migration. Dev Biol. 2003;261(1):251-267.

28. Rama N, et al. Slit2 signaling through Robo1 and Robo2 is required for retinal neovascularization.
Nat Med. 2015;21(5):483-491.

29. Wang B, et al. Induction of tumor angiogenesis by Slit-Robo signaling and inhibition of cancer growth by blocking Robo activity. Cancer Cell. 2003;4(1):19-29.

30. Larrivée B, Freitas C, Suchting S, Brunet I, Eichmann A. Guidance of vascular development: lessons from the nervous system. Circ Res. 2009;104(4):428-441.

31. Ramkhelawon B, et al. Netrin-1 promotes adipose tissue macrophage retention and insulin resistance in obesity. Nat Med. 2014;20(4):377-384.

32. Rosenberger $P$, et al. Hypoxia-inducible factor-dependent induction of netrin-1 dampens inflammation caused by hypoxia. Nat Immunol. 2009;10(2):195-202.

33. Kennedy TE, Serafini T, de la Torre JR, Tessier-Lavigne M. Netrins are diffusible chemotropic factors for commissural axons in the embryonic spinal cord. Cell. 1994;78(3):425-435.

34. Serafini T, Kennedy TE, Galko MJ, Mirzayan $\mathrm{C}$, Jessell TM, Tessier-Lavigne M. The netrins define a family of axon outgrowth-promoting proteins homologous to C. elegans UNC-6. Cell. 1994;78(3):409-424.

35. Moore SW, Tessier-Lavigne M, Kennedy TE. Netrins and their receptors. Adv Exp Med Biol. 2007;621:17-31.

36. Höpker VH, Shewan D, Tessier-Lavigne M, Poo $\mathrm{M}$, Holt C. Growth-cone attraction to netrin-1 is converted to repulsion by laminin-1. Nature. 1999;401(6748):69-73.

37. Lai Wing Sun K, Correia JP, Kennedy TE. Netrins: versatile extracellular cues with diverse functions. Development. 2011;138(11):2153-2169.

38. Dickson BJ, Keleman K. Netrins. Curr Biol. 2002;12(5):R154-R155.

39. Bányai L, Patthy L. The NTR module: domains of netrins, secreted frizzled related proteins, and type I procollagen C-proteinase enhancer protein are homologous with tissue inhibitors of metalloproteases. Protein Sci. 1999;8(8):1636-1642.

40. Navaratna D, McGuire PG, Menicucci G, Das A. Proteolytic degradation of VE-cadherin alters the blood-retinal barrier in diabetes. Diabetes. 2007;56(9):2380-2387.

41. Levin JI, et al. The discovery of anthranilic acidbased MMP inhibitors. Part 2: SAR of the 5-position and P1(1) groups. Bioorg Med Chem Lett. 2001;11(16):2189-2192.

42. Koch AW, et al. Robo4 maintains vessel integrity and inhibits angiogenesis by interacting with UNC5B. Dev Cell. 2011;20(1):33-46.

43. Sapieha PS, Peltier M, Rendahl KG, Manning WC, Di Polo A. Fibroblast growth factor-2 gene delivery stimulates axon growth by adult retinal ganglion cells after acute optic nerve injury. $\mathrm{Mol}$ Cell Neurosci. 2003;24(3):656-672.

44. Sapieha P. Eyeing central neurons in vascular growth and reparative angiogenesis. Blood. 2012;120(11):2182-2194.

45. Podjaski C, et al. Netrin 1 regulates blood-brain barrier function and neuroinflammation. Brain. 2015;138(pt 6):1598-1612.
46. Bin JM, et al. Full-length and fragmented netrin-1 in multiple sclerosis plaques are inhibitors of oligodendrocyte precursor cell migration. Am J Pathol. 2013;183(3):673-680.

47. Jarjour AA, et al. Maintenance of axo-oligodendroglial paranodal junctions requires DCC and netrin-1. J Neurosci. 2008;28(43):11003-11014.

48. Tepavčević V, et al. Early netrin-1 expression impairs central nervous system remyelination. Ann Neurol. 2014;76(2):252-268.

49. Oksala N, et al. Association of neuroimmune guidance cue netrin- 1 and its chemorepulsive receptor UNC5B with atherosclerotic plaque expression signatures and stability in human(s): Tampere Vascular Study (TVS). Circ Cardiovasc Genet. 2013;6(6):579-587.

50. Liu D, Xia XB, Xu XL, Tian XF, Shang L. Role of unc $5 \mathrm{~b}$ in retinal neovascularization in mice with oxygen-induced retinopathy. Int JOphthalmol. 2011;4(2):137-142.

51. Van Lint P, Libert C. Chemokine and cytokine processing by matrix metalloproteinases and its effect on leukocyte migration and inflammation. JLeukoc Biol. 2007;82(6):1375-1381.

52. Lee S, Jilani SM, Nikolova GV, Carpizo D, Iruela-Arispe ML. Processing of VEGF-A by matrix metalloproteinases regulates bioavailability and vascular patterning in tumors. JCell Biol. 2005;169(4):681-691.

53. Shipp EL, Hsieh-Wilson LC. Profiling the sulfation specificities of glycosaminoglycan interactions with growth factors and chemotactic proteins using microarrays. Chem Biol. 2007;14(2):195-208.

54. Trexler M, Bányai L, Patthy L. A human protein containing multiple types of proteaseinhibitory modules. Proc Natl Acad Sci U S A. 2001;98(7):3705-3709.

55. Kowluru RA, Zhong Q, Santos JM. Matrix metalloproteinases in diabetic retinopathy: potential role of MMP-9. Expert Opin Investig Drugs. 2012;21(6):797-805.

56. Coussens LM, Fingleton B, Matrisian LM. Matrix metalloproteinase inhibitors and cancer: trials and tribulations. Science. 2002;295(5564):2387-2392.

57. Vandenbroucke RE, Libert C. Is there new hope for therapeutic matrix metalloproteinase inhibition?. Nat Rev Drug Discov. 2014;13(12):904-927.

58. Kaufmann KW, Lemmon GH, Deluca SL, Sheehan JH, Meiler J. Practically useful: what the Rosetta protein modeling suite can do for you. Biochemistry. 2010;49(14):2987-2998.

59. Kennedy TE, Wang H, Marshall W, Tessier-Lavigne M. Axon guidance by diffusible chemoattractants: a gradient of netrin protein in the developing spinal cord. J Neurosci. 2006;26(34):8866-8874.

60. Gonzalez HL, Carmichael N, Dostrovsky JO, Charlton MP. Evaluation of the time course of plasma extravasation in the skin by digital image analysis. J Pain. 2005;6(10):681-688.

61. Smith LE, et al. Oxygen-induced retinopathy in the mouse. Invest Ophthalmol Vis Sci. 1994;35(1):101-111. 